\title{
NeoMinkowskian Cosmological Black Hole, Poincaré's Gravific Electron and Density of CBR
}

\author{
Yves Pierseaux \\ ULB (iihe), Brussels, Belgium \\ Email:ypiersea@ulb.ac.be
}

How to cite this paper: Pierseaux, Y. (2020) NeoMinkowskian Cosmological Black Hole, Poincaré's Gravific Electron and Density of CBR. Journal of Modern Physics, 11, 237-280.

https://doi.org/10.4236/jmp.2020.112015

Received: December 17, 2019

Accepted: February 17, 2020

Published: February 20, 2020

Copyright (๑) 2020 by author(s) and Scientific Research Publishing Inc. This work is licensed under the Creative Commons Attribution International License (CC BY 4.0).

http://creativecommons.org/licenses/by/4.0/

\section{(c) (i) Open Access}

\begin{abstract}
In the previous paper (JMP 2014) we showed that there exists a NeoMinkowskian Gravitational Expanding Solution of GR (General Relativity) with CC (Cosmological Constant). We prove now that NeoMinkowskian Vacuum (non-baryonic Fluid), with gravitational (first) density (dark energy) and gravitational waves (at light speed), corresponds to the Gravitation Field of a Cosmological Black Hole $(\mathrm{CBH})$. The latter predicts furthermore a basic emission of Radiation (CBR) from Hubble spherical singular Horizon to the inside of $\mathrm{CBH}$ (unlike Hawking's emission) at an initial singular time. Our solution is then compatible with a well-tempered Big Bang and Expanding Universe (Escher's Figure, see Penrose, §3) but incompatible with inflation. The latter is based on Hypothesis of a so-called Planck's particle (Lemaitre's primitive atom) characterized by a so-called Planck length. We prove that we can short-circuit this unstable particle with a stable cosmological Poincaré's electron with gravific pressure. It is well known that electron is a stranger in usual Minkowskian vacuum (dixit Einstein). The stranger electron can be perfectly integrated in NeoMinkowskian Radiation fluid and then also (with its mass, charge and wavelength) in (second density of) CBR. Everything happens as if the leptonic mass of the electron were induced by our cosmological field. The unexpected cosmological model proposed here is the only one that predicts numerical values of (second) density and temperature of $C B R$ very close to the observed $(C O B E)$ values.
\end{abstract}

\section{Keywords}

Cosmological Constant, General Relativity, Minkowskian Metric, Cosmological Black Hole, Tachyons, Hyperbolic Horizon, Density of Vacuum, Density of CBR, Poincaré's Gravitational Waves, Poincaré's Electron, De Broglie's Wave Electrodynamics, De Broglie's Subquantum Substratum 


\section{Introduction: NeoMinkowskian Gravitational Vacuum as Solution of GR with CC}

Let's summarize first how we come to an unexpected Gravitational NeoMinkowskian Expanding Vacuum which “looks like” de Sitter's Expanding Vacuum [1] [2].

(1) Gravitational Density of NeoMinkowskian Vacuum

Let us consider Einstein's equation of General Relativity $(G R)$ with Cosmological Constant $(C C) \Lambda$ and Perfect Fluid $T_{\mu v} \equiv(p+\rho) \frac{u_{\mu} u_{v}}{c^{2}}-p g_{\mu v}$ (standard notations: energy density $\rho$, pressure $p$ and 4-velocity $\left.u_{\mu} u_{v}\right)$ :

$$
G_{\mu v}+\Lambda g_{\mu v}=\chi T_{\mu v}=\frac{8 \pi G}{c^{4}} T_{\mu v}=\frac{8 \pi G}{c^{4}}\left[(p+\rho) \frac{u_{\mu} u_{v}}{c^{2}}-p g_{\mu v}\right]
$$

Let us introduce Minkowskian Metric (MM) $g_{\mu v}=\eta_{\mu v}$ in (0) with therefore cancellation of Einstein's curvature tensor $G_{\mu v}=0$ :

$$
G_{\mu v}=0 \Rightarrow \Lambda \eta_{\mu v}=\frac{8 \pi G}{c^{4}} T_{\mu v}=\frac{8 \pi G}{c^{4}}\left[(p+\rho) \frac{u_{\mu} u_{v}}{c^{2}}-p \eta_{\mu v}\right]
$$

A non-trivial NeoMinkowskian Solution with $\rho=-p \quad(\rho+p=0)$ is then:

$$
T_{\mu v}^{V A C U U M}=\rho \eta_{\mu v}=\frac{\Lambda c^{4}}{8 \pi G} \eta_{\mu v}
$$

The density of VACUUM, simulated by a Non-Baryonic ( $G_{\mu v}=0$ ) NeoMinkowskian Fluid, depends therefore on the Gravitational Constant (see 14):

$$
\rho+p=0 \Rightarrow \rho=\frac{\Lambda c^{4}}{8 \pi G}=\rho_{\Lambda}
$$

This Gravitational NEO-Minkowskian Vacuum (GMV) does not correspond to the usual ElectroMagnetic Vacuum (EMV) (with permittivity, permeability, impedance...) of the standard Minkowskian Vacuum of Special Relativity $(S R)$. We reject here any attempt of putsch ${ }^{1}$ that consists in canceling (a priori) this gravitational density (1bis): $p=\rho=0$.

We are looking first for the determination of the global gravitational field ( $\$ 1)$ which corresponds to this density and then for Poincaré's Gravific Waves (in the framework of Lorentz Transformation, (LT, see $\S 1-3,8)$. The operation $p=\rho=0$ is characteristic of de Sitter's Vacuum whose metric ( $d S M)$ and not that of Minkowski ( $M M \neq d S M$ ). In basic equation (0), NeoMinkowskian Vacuum is based on cancellation $(\rho+p=0)$ of Einstein's Tensor $\left(G_{\mu v}=0\right)$ whilst deSitterian Vacuum is based on cancellation ( $p=\rho=0$ ) of Tensor of Perfect Fluid $\left(T_{\mu v}=0\right)$ :

$$
G_{\mu v}+\Lambda g_{\mu v}=0
$$

At first glance, these two approaches to Vacuum (0- $d S M$ and $0-M M)$ are very far apart. We first show (in introduction, see also JMP previous paper) that they are indeed very close (6).

${ }^{1}$ Given that Einstein's (local) curvature tensor $G_{\mu v}=0$. is canceled, it is generally admitted that Global NeoMinkowskian space is flat and so the basic density (1bis) is canceled. Let us note that CC dimensionally corresponds to a global (negative) curvature, see \$3-3-2). 
The only way to develop the role of $C C$ in NeoMinkowskian Vacuum is to start from that of $C C$ in Riemanian Vacuum.

(2) Cosmological Constant (CC) in Expanding Riemannian Vacuum

In cosmological literature we never find (1) but we always find (anti-1) with Riemannian Metric $(R M) g_{\mu \nu}\left(G_{\mu \nu} \neq 0\right)$ :

$$
T_{\mu v}^{\text {DarkEnergy }}=\frac{\Lambda c^{4}}{8 \pi G} g_{\mu v} \Rightarrow \rho+p=0
$$

The cosmological term $\Lambda g_{\mu \nu}$ (first member) is associated, by the cosmologists, with the density term (second member) $\frac{8 \pi G}{c^{4}} \rho g_{\mu v}$. This so-called "component of vacuum" (Dark Energy) is then associate to $\rho+p=0$ (next to other components: radiation component, matter component...).

In order to avoid the fateful. $G_{\mu v}=0 \Rightarrow g_{\mu v}=\eta_{\mu v}$ (1), the strategy of cosmologists consists of multiplying (0) into several equations (phases or periods): one equation for matter, one equation for radiation, one equation for curvature, one equation for dark energy (before the matter and then $G_{\mu \nu}=0$ )... (each equation corresponding to a period of the inflation).

We have in our strategy "one and only one equation (0)" with one and only one solution (1 bis, 9,12$)^{2}$.

In order to find the characteristics of Dark Energy, cosmologists develop the thermodynamics underlying the relationship $\rho+p=0$ :

$\rho+p=0 \Rightarrow H=U+p V=0 \Rightarrow \mathrm{d} H=\mathrm{d} U+p \mathrm{~d} V=0 \Rightarrow \rho+p=0 \quad$ (1-\&-anti-1)

Local transformation $\rho+p=0$ leads by integration to a global Volume $U=\rho V$. By differentiation $\mathrm{d} U+p \mathrm{~d} V=0$ we return to the local starting point $\rho+p=0 \quad\left(\frac{\mathrm{d} U}{\mathrm{~d} V}=\rho\right)$ if and only if $\mathrm{d} V \neq 0$.

The variable volume results from a well known property of Enthalpy $(H=U+p V$ isentropic $\mathrm{d} S=0$ and non isochoric variation $\mathrm{d} V \neq 0)$.

Nothing prevents then introducing a variability over time and therefore a growing energy in an expanding empty universe:

$$
\rho+p=0 \Rightarrow U(t)=\rho V(t)
$$

which can be developed $\mathrm{d} V \neq 0$ on the basis of any metric $g_{\mu v}$ included $\eta_{\mu \nu}$. This is our starting point (2) with a very binding determination of the metric: $g_{\mu v}=\eta_{\mu v}$ (as we will see it, NeoMinkowskian constraint of inaccessible singular $(<c$ or $>c)$ velocity is very strong).

(3) Cosmological Constant (CC) in Exponential Expanding NeoMinkowskian Vacuum

The first Minkowskian constraint on (2), in a pseudoEuclidean space-time $4 D$ framework, involves a spherical (Euclidean) $3 D$ symmetry:

$V(t)=\frac{4}{3} \pi R^{3}(t) \Rightarrow \dot{V}(t)=\frac{4}{3} \pi 3 R^{2}(t) \dot{R}(t) \Rightarrow \frac{\dot{U}(t)}{U(t)}=\frac{\dot{V}(t)}{V(t)}=3 \frac{\dot{R}(t)}{R(t)}$

The non-static NeoMinkowskian $G M V$ has to be treated with a radial scale

${ }^{2}$ We will see ( $\left.\$ 5\right)$ that another density that corresponds (see $\$ 4$ ) to the same solution. 
factor $R(t)$ and a radial velocity $\dot{R}(t)$ and therefore with a radial ratio $\frac{\dot{R}(t)}{R(t)}$. Given that we have indifferently a constant density of energy or a constant density of mass $\rho_{M}=\frac{\rho}{c^{2}}=\rho_{\Lambda}$ as well, NeoMinkowskian spherical symmetry imposes Einstein's equivalence relation between (dark) pseudo-energy $U(t)$ and (non-baryonic) pseudo-mass $M(t)$ :

$$
M(t) c^{2}=U(t)=\frac{4}{3} \pi \rho R^{3}(t)
$$

We have thus a global variable pseudo-mass a $M(t)=\frac{4}{3} \pi \frac{\rho}{c^{2}} R^{3}(t)$ and a local constant pseudo-mass density $\frac{\rho}{c^{2}}$ (in radial Friedman's equation it is usually the opposite situation).

Nothing prevents a priori that this pseudo-mass from being sensitive to Newton law of gravitation.

On one hand a static model with potential energy $E_{P}=-\frac{G M}{R}$ is excluded because unstable (collapse).

On the other hand a dynamical model, based on an equilibrium between kinetics energy $E_{c}$ and potential energy $E_{P}$, is possible:

$$
\frac{1}{2} \dot{R}(t)^{2}-\frac{G M(t)}{R(t)}=\frac{1}{2} \dot{R}(t)^{2}-\frac{4}{3} \pi G \frac{\rho}{c^{2}} R(t)^{2}=0
$$

(YP12 with common numbering in the French and English version of our previous research [1] [2] $)^{3}$. We simplified with a Non-Zero enigmatic Non-Baryonic $\left(N B, G_{\mu v}=0\right)$ (micro) mass $m_{N B}$ :

$$
\frac{1}{2} m_{N B} \dot{R}(t)^{2}-\frac{G M(t) m_{N B}}{R(t)}=0
$$

We can then determine the desired ratio (2 bis):

$$
\frac{\dot{R}(t)}{R(t)}=\sqrt{\frac{1}{3} \frac{8 \pi G}{c^{2}} \rho}=c \sqrt{\frac{\Lambda}{3}}=H_{\Lambda}
$$

From $\rho+p=0$ we return to $C C$ with $^{4} \Lambda=\frac{8 \pi G \rho}{c^{4}}$.

That means that our dynamical equation $(4$, YP12) is an alternative to historical Einstein's determination of constant $\chi$ in (0) with weak Newtonian Gra-

${ }^{3} \mathrm{~A}$ dynamic fatal objection seems to be however formulated here at this stage: Our model $(\mathrm{YP} 12,4)$ is neither Minkowskian nor NeoMinkowskian because gravitational law with especially kinetics energy would non relativistic. That would be true for a material (baryonic) point ( $\S 3$ ). We urge the reader to be cautious (and patient, $\$ 3$ ) because no one has tried until now to apply scalar (temporal) Newton law of gravitation to a space point (we are in the framework of $G R$ ) that is to say a non-baryonic (non-material) pseudo-mass. Nobody really knows (at this stage) if this law (4) is incompatible or not with $M M$.

${ }^{4}$ With $\Lambda=0 \quad$ we return to static Minkowskian usual solution $\frac{\dot{R}(t)}{R(t)}=0$. 
vitation Field (metric $g_{\mu v}=\eta_{\mu v}+h_{\mu v}$ ). Indeed from (0) $\Lambda \eta_{\mu v}=\chi T_{\mu v}=\chi \rho \eta_{\mu v}$ we have $\rho=\frac{\Lambda}{\chi}$ and from (5) $\chi=\frac{\Lambda}{\rho}=\frac{8 \pi G}{c^{4}}$. The determination of constant $\chi$ with NeoMinkowskian gravitation field (4) is then much more direct than that with the weak gravitation field.

On the basis of YP12 we rediscover gravitational (critical) density (1-bis) in function of (measurable) Hubble's constant (see numerical value in annex 1 with Gauss cgs-units):

$$
\rho_{M}=\frac{\rho}{c^{2}}=\rho_{\Lambda}=\frac{3 H_{\Lambda}^{2}}{8 \pi G}
$$

Our non-usual solution consists therefore in an Exponential Expanding (EE) of Global Vacuum with a Hubble (measurable) Constant which defines the kinematics underlying the future gravitational dynamics, $\S 1$ ):

$$
R(t)=R(0) \mathrm{e}^{H_{\Lambda} t}, \quad \dot{R}(t)=\dot{R}(0) \mathrm{e}^{H_{\Lambda} t} .
$$

It was at this point that we had arrived in our previous work [1] [2], (6) in keeping with recent observations of an accelerating (see 10) expanding universe [3].

Nevertheless the originality of our NeoMinkowskian $E E$ of Vacuum, very close to deSitterian $E E$ of Vacuum (with zero density, §1-4), did not seem obvious yet.

So the binding singularity of light velocity, underlying any Minkowskian (or NeoMinkowskian) solution, does not appear in (6). Except if we impose a structural speed of NeoMinkowskian space-time $\dot{R}(0)=c$ in Initial Conditions (IC).

This is the reason why we suggest to examine first, on a kinematic point of view, the contrast "deSitterian $I C(\S 1-1)$ versus NeoMinkowskian $I C$ " (§1-2).

\section{1. [TACHYON] Only Can Escape (to the Outside) from Cosmological Horizon of NeoMinkowskian Black Hole}

The structure of the paper will follow 3-partition of NeoMinkowskian space-time: Tachyon $(v>c)$ $\S$, Photon $(v=c) \S 2$, Bradyon $(v<c) \S 3$.

\subsection{Undetermined Initial Conditions (IC) in deSitterian EE (Inflation)}

Let's take a look at deSitterian solution that consists of canceling the second member: $T_{\mu \nu}=0$ or $p=\rho=0$ in (0):

$$
0=G_{\mu v}+\Lambda g_{\mu v}=\frac{\dot{a}^{2}(t)}{a^{2}(t)}-\frac{\Lambda}{3}
$$

On the left we write Einstein's equation (0) and on the right radial Friedman's equation ${ }^{5}$ with Robertson-Walker's metric. The latter is based on two factors (scale factor $a(t)$ and curvature factor $K$ ).

${ }^{5}$ Let us note that NeoMinkowskian solution (1 or 5 ) is also a solution of Friedman's equation with $a(t)=1 \quad(K=0)$. The scale factor disappears. Everything seems static. Unless the scale factor is hidden in a scale hyperbola ( $\$ 2-3)$. We note that our model does not correspond to pseudo-hyperbolic value of parameter $K=-1$ in $R W$ (see $\S 3-3$ ). 
The simplest deSitterian solution is then Parabolic $(K=0)$ Vacuum $(d S V)$ without matter $\rho=0$, without structural light velocity $c$ and even without gravitational constant $G$ : the factor $\frac{8 \pi G}{c^{4}}$ being eliminated from both Einstein's and Friedman's equation (Hubble's constant is the same (true constant) connected with $C C$ in both cases $c \sqrt{\frac{\Lambda}{3}}=H_{\Lambda}$ ):

$$
\frac{\dot{a}(t)}{a(t)}=c \sqrt{\frac{\Lambda}{3}}=H_{\Lambda} \Rightarrow a(t)=a(0) \mathrm{e}^{H_{\Lambda} t}, \dot{a}(t)=\dot{a}(0) \mathrm{e}^{H_{\Lambda} t}
$$

We propose the numbering "anti" 6 for three reasons:

1) deSitterian Hubble constant is not connected with density $(\rho=0)$ and so to a determined gravitational dynamic ( 5 bis).

2) deSitterian $I C$ are undetermined, $a(0), \dot{a}(0)$, for the radial distance $a(0)$ and radial velocity $\dot{a}(0)$ as well. We can choose to introduce the radius of the so-called Planck's particle (Planck's length, see annex 1), Poincaré's radius of electron, Bohr's radius of atom, Erathostène's radius of Earth, diameter of solar system, diameter of Milky Way as well.

3) In de Sitter's model ( $d S V$ ) there is no singular IC (Horizon). It means that the spherical surface (for example the radius of Hubble) $a(0)$ is continually moving (instantly) at the time $t=0$ with velocity $\dot{a}(0)$ (for example $(\dot{a}(0)=c)$.

\subsection{Determined IC in NeoMinkowskian EE: From Singular Velocity to Singular Cosmological Horizon}

If we set the light speed $\dot{R}(0)=c$ in (6) to an initial time, say zero $(t=0)$, then this singular constant speed immediately implies, with $H_{\Lambda} R_{H}=c$ a singular constant Radius $R(0)=R_{H}=\sqrt{\frac{3}{\Lambda}}$. ( $H$ for Horizon and for Hubble).

The only physical (and logical) consistent interpretation of this double discontinuity (Horizon) involves a singular EMISSION of light, at the (fixed) speed c, from a singular spherical surface Horizon whose Radius $R(0)=R_{H}$ is fixed in $t=0$ (unlike deSitterian continuity).

Unlike de Sitter's initial radius, NeoMinkowskian initial radius $\left(R(0)=R_{H}\right)$ must be the Horizon of Hubble (binding determination induced by $C C$ ).

Unlike usual Minkowskian theory, NeoMinkowskian theory (6) has a DOUBLE HORIZON $\left(c, R_{H}\right)$ : Horizon of Velocity and Horizon of Space.

The complete explicit solution (6), that takes into account imposed IC in $t=0 \quad\left(R(0)=c\right.$ and $\left.R(0)=R_{H}\right)$ is then:

$\frac{\dot{R}_{\text {Tachy }}(t)}{R_{\text {Tachy }}(t)}=H_{\Lambda}=\sqrt{\frac{8 \pi G \rho}{3}} \Rightarrow R(t)=R_{H} \mathrm{e}^{H_{\Lambda} t}, \dot{R}(t)=c \mathrm{e}^{H_{\Lambda} t} \Rightarrow c=H_{\Lambda} R_{H}$

For a time later $t>0$ we will have therefore supra-luminous speeds $\dot{R}(t)>c$ of TACHYONS (the first member of Feinberg's trio) that move away from the 
singular Horizon $R(0)=R_{H}$ We have a law of Hubble but for tachyons $\dot{R}(t)=H_{\Lambda} R(t)$ ! (for bradyons see, 19 bis).

Our tachyonic model of an expanding Universe fits well in the framework of $G R$ where nothing forbids that the speed of a space point is greater than a singular (light) speed. And therefore nothing forbids that the speed of a space point is ALWAYS greater than a singular (light) speed.

Given that the velocity MUST be super-luminous $\dot{R}(t)>c$ for tachyons if and only if we have no negative time $t>0$ the original time $t=0$ is then a (true) singularity.

The latter must logically correspond to a finite time $T_{H}=H_{\Lambda}^{-1}$ in the same way that we have a finite space Horizon. Our NeoMinkowskian model with a finite time is then compatible with a Big Bang.

Given that the Hubble Horizon $R_{H}$ is given at initial time $t=0$, our NeoMinkowskian model is NOT compatible with (deSitterian) inflation of a supposed "Planck's particle" that delete $\left(R_{H} \rightarrow l_{P} \rightarrow 0\right)$ Hubble's Horizon".

The paper could stop here: We deduce the first expanding $G R$-Solution with STRICT NeoMinkowskian inequality $\left(R(t)>R_{H}-\dot{R}(t)>c\right)$ : Only tachyons can escape from the Horizon.

But what are they escaping from? ( $§ 1-3)$

Unlike deSitterian zero density, NeoMinkowskian non-zero gravitational density (1bis) defines a gravitational field (constant $G$ ). What is this searched gravitational field $G M V(\S 1-3)$ ?

The paper could then not stop here because we are looking for a full description (kinematic and dynamic) of NeoMinkowskian space-time (tachyon, photon, bradyon).

\subsection{NeoMinkowskian Cosmological Black Hole ( $\mathrm{CBH})$ and Poincaré's Gravific Waves}

So far we have developed the (Radial) Kinematic (6, 6 bis) aspect of the NeoMinkowskian solution. Let's now develop the Gravific (Scalar) Dynamic aspect. In order to do that, let us now introduce our Kinematic NeoMinkowskian $I C$ in Dynamic $Y P 12(5)$ at singular time $t=0$ :

$$
\frac{1}{2} \dot{R}(t)^{2}-\frac{G M(t)}{R(t)}=\frac{1}{2} \dot{R}(0)^{2}-\frac{G M(0)}{R(0)}=\frac{1}{2} c^{2}-\frac{G M_{H}}{R_{H}}=0
$$

With a new gravific dynamic IC $M_{H}=\frac{4}{3} \pi \frac{\rho}{C^{2}} R_{H}^{3}$ we deduce the threshold escape speed:

$$
c^{2}=\frac{2 G M_{H}}{R_{H}}
$$

An Horizon from which a PHOTON cannot escape (only Tachyon can es-

${ }^{6}$ Since there is neither observed expansion of the galaxies themselves nor the solar (or atomic) system itself, the existence a priori of a Hyperbolic Horizon is then consistent with current observations $(\S 3-3)$. 
cape) is by definition a Schwarzshild s Horizon of a BLACK HOLE.

More precisely: A Cosmological Black Hole $(C B H)$ whose Universal Schwarzshild's Horizon is Hubble's Horizon.

In parallel with a 3-partition of the speed space (tachyons, photons and bradyons), our $C B H$ involves a 3-partition of space itself (out, on and in).

THE searched GRAVITATIONAL FIELD $G M V$ which corresponds to gravitational density $(1,1$ bis) is given by A UNIVERSAL NEOMINKOWSKIAN BLACK HOLE $(\mathrm{CBH})$.

Underlying Minkowskian Metric $M M$ must be then written as follows:

$$
\mathrm{d} s^{2}=\mathrm{d} r^{2}-c^{2} \mathrm{~d} t^{2}=\mathrm{d} r^{2}-\frac{2 G M_{H}}{R_{H}} \mathrm{~d} t^{2}
$$

It was enough to think about it!

According to $G R$ transformations of coordinates with $M M$ are LINEAR LORENTZ TRANSFORMATION ( $L T)$ (see $§ 2-3,18$ scale hyperboles).

On such grounds it is no longer possible to claim that $M M$ is incompatible with gravitational density (1 bis), with gravitational field in vacuum (8) and then also with possible existence of GRAVITATIONAL WAVES at light velocity.

This is exactly Poincaré's position in 1905 with his "Gravific Waves": "Quelles modifications elle [la transformation de Lorentz] nous obligerait? apporter aux lois de la gravitation. Cest ce que jai cherché à déterminer. $\rho$ ai été conduit à supposer que la propag ation de la gravitation [ondes gravifique dixit Poincare] n" est pas instantanée mais qu' elle se fait à la vitesse de la lumière" (introduction) [4].

So we are led to rehabilitate Poincaré's work on $L T$ in 1905 but on the basis... of Einstein's $G R$ in 1916 (with $C C$ 1917). So there is no polemical intention on our part (see annex 2).

Formula (8) looks like Laplace's (Mitchell's) non-relativistic formula of static Stellar Black Hole $(S B H)$. There is neither singular escape velocity $\left(v^{2}=c^{2}=\frac{2 G M_{S}}{R_{S}}\right)$ nor singular Horizon in non-relativistic Laplace's approach.

IN SUMMARY: The only way to make relativistic Laplace's formula (9 with 10) consists in claiming that alone tachyonic points can escape from event Horizon. But we need then a gravitational field $(C B H)$ which does not exist in usual $S R$. YP-12 involves that the desired $G M V$ is then $C B H$ in NeoMinkowskian global solution (with a negative hyperbolic curvature, see $\S 3$ ).

At this stage our NeoMinkowskian Black Hole $(C B H)$ seems very close to that

\footnotetext{
${ }^{7}$ We purposely used the term GRAVIFIC WAVE used by Poincaré in 1905. Laplace considered that a velocity CAN be super-luminous: "La gravitation se déplace au moins 300 fois plus vite que la lumière. Poincaré criticizes Laplace in 1905 by proposing that the speed of a gravitational wave must be the limit (singular) speed of light on the basis of Lorentz Transformation (LT)". Poincaré's position on gravific waves is non orthodox because $S R$ is reputed without gravitation, without density (1 bis, the putsch note 1) and then without gravific waves, note 8 ).

He shows also (in $\S 6$ of the same paper) that ELECTRON undergoes a GRAVIFIC pressure ( $\S$ $5-2)$ in the framework of $L T$. Thanks to NeoMinkowskian approach, we will be able to synthesize the G-wave and the G-pressure ( §5-2).
} 
of cosmology of FLAT Universe (Tatum $\left.{ }^{8},[5]\right)$.

Let us precise in order to complete the dynamic that we have also an acceleration $\ddot{R}(t)$ deduced with a second derivation:

$\ddot{R}(t)-H_{\Lambda}^{2} R(t)=0 \Rightarrow q_{\Lambda}=-\frac{R(t) \ddot{R}(t)}{\dot{R}(t)^{2}}=-1 \Rightarrow \frac{R(0) \ddot{R}(0)}{\dot{R}(0)^{2}}=\frac{R_{H} \alpha_{M}}{c^{2}}=1$

with a new basic invariant constant $\alpha_{M}$ determined in $t=0 . Y P$-12 introduces a global singular pseudo-mass $M_{H}$ of $C B H$ (singular dark Energy $U_{H}=M_{H} C^{2}$ which might seem unrealistic if it did not fit with a singular acceleration $\alpha_{M}$. This basic acceleration $\alpha_{M}$ cannot be applied to the constant singular velocity of emission of photon but can be applied to tachyons (centripetal $\alpha_{M}$ ) and to bradyons (centrifugal $\alpha_{M}$ ) as well ( $\S 3.3$ ).

So we have to continue our path from very formal and abstract maximal (centripetal) acceleration for tachyon, to a very physical and concrete minimal (centrifugal) acceleration for bradyon (25) ${ }^{9}$. We will show that this hyperbolic acceleration is a universal invariant ( $\$ 3-3)$ and therefore all the above dynamic gravitational equations are perfectly relativistic in NeoMinkowskian meaning.

Finally we check the consistence of our approach with the deduction of the "second" usual cosmological parameter (Hubble $H_{\Lambda}$ and acceleration $q_{\Lambda}$ ):

$$
q_{\Lambda}=\frac{\rho}{p}=-1 \rightleftarrows \alpha_{M}=H_{\Lambda} c=\frac{2 G M_{H}}{R_{H}^{2}}
$$

In truth they are here both sides of the same coin. Let us insist on the necessity of not confusing $q_{\Lambda}=-1$ with $K=-1$. In the first case it is a reference to a basic global hyperbolic motion $\left(q_{\Lambda}=-1\right)$ with constant acceleration $\alpha_{M}$ whilst in the second case it is a reference to the pseudo-hyperbolic value of local curvature $(K=-1)$ parameter in $R W$ metric (note 5).

By conferring a positive role to an essential component (tachyons) of the NeoMinkowskian non-baryonic framework $\left(G_{\mu \nu}=0\right)$ the unique solution (1 or 9) can henceforth be written:

$$
T_{\mu \nu}^{\text {VACUUM }}=\frac{\Lambda c^{4}}{8 \pi G} \eta_{\mu \nu}^{-}=\rho_{\Lambda}\left(\begin{array}{cccc}
-1 & 0 & 0 & 0 \\
0 & 1 & 0 & 0 \\
0 & 0 & 1 & 0 \\
0 & 0 & 0 & 1
\end{array}\right)
$$

Supra-luminous speed of tachyon is perfectly allowed in $G R$ for space-points.

\footnotetext{
${ }^{8} \mathrm{We}$ have somehow established a theoretical horizon for formulas of flat universe ( $K=0$ in $R W$, see note 5). The problem is that this Horizon must be Hyperbolic (see $\$ 3$ ).

${ }^{9}$ Outside the $C B H$ (minimal light velocity) we have a minimal $R_{H}$ coupled with a maximal (centripetal) $\alpha_{M}$. Inside the $C B H$ we will have exactly the opposite (minimal centrifugal $\alpha_{M} \quad \S 3-3$ ). The objection that the acceleration cannot be a relativistic invariant is inadmissible because both theories are not in competition at this stage. The question will arise when the bradyon that matches to tachyon will be defined. To reassure the reader, we will have ( $\$ 3)$ STRICT inequalities in both cases (tachyons and bradyons) but in inverted sense $\left(R(t)>R_{H}-\dot{R}(t)>C\right.$ and $\left.r(t)<R_{H}-v(t)<c\right)$. It will be solve with hyperbolic acceleration which is a relativistic invariant (( \$3-3). IDEM for Newton's laws (dynamic and gravitation as well).
} 
Our $G R$-solution (1-1bis-1-ter) is always valid whatever the value of velocity (> $=$ and $<$ ) because the basic operation $\rho+p=0$ makes disappear the factor $(p+\rho) \frac{u_{\mu} u_{v}}{c^{2}}$ with 4 -velocities (the temporal component is here -1 in 00$)$. The positive role of tachyons induces internal density of dark energy $\left(\rho_{\Lambda}=\frac{3 M_{H} c^{2}}{4 \pi R_{H}^{3}}\right)$. Is this the only possible density? Is there another density defined by

$\eta_{\mu \nu}^{+}=\left(\begin{array}{cccc}1 & 0 & 0 & 0 \\ 0 & -1 & 0 & 0 \\ 0 & 0 & -1 & 0 \\ 0 & 0 & 0 & -1\end{array}\right)$ (see $\left.\S 3-3\right)$ which is not directly the unique solution $(1$, 12) but which is coupled ( $\S 4$ see the ratio anti-32 \& $\S 5)$ with the density (1-bis, 1 -ter)?

\subsection{A Cosmological Hidden Non-Baryonic Micro-Mass?}

Both Newton s laws (Gravitation and Dynamic) are coupled $(t=0)$ :

$$
F_{G}=\frac{c^{4}}{2 G}=M_{H} \alpha_{M}=M_{H} \frac{2 G M_{H}}{R_{H}^{2}}
$$

(density 1 bis can be write $\rho_{\Lambda}=\frac{3 F_{G}}{4 \pi R_{H}^{2}}$ ). We have not only a Non-Baryonic $(N B)$ Macro-Mass $M_{H}$ but also a $N B$ micro-mass $m_{N B}$ (4 bis):

$$
\frac{1}{2} m_{N B} c^{2}-\frac{G M_{H} m_{N B}}{R_{H}}=0 \quad(Y P 12) \quad \Rightarrow m_{N B} c^{2} R_{H}=\frac{2 G M_{H} m_{N B}}{R_{H}^{2}}
$$

We see that we have next to a Macro Force (13) a Newton's law of dynamic a micro force (14):

$$
f_{G}=m_{N B} \alpha_{M}=\frac{2 G M_{H} m_{N B}}{R_{H}^{2}}=2 m_{N B} G \frac{M_{H}}{R_{H}^{2}}
$$

If $F_{G}$ (13) is determined, $f_{G}(14)$ is, at this stage, undetermined ( $N B$ micro-mass will be precise in $\S 4$ ). This will be a very important point for Poincaré’s "dynamic of electron" $(\S 5)$ with a gravitational force $\left(m_{N B}=m_{e}\right.$ ?).

\subsection{From Static Stellar Black Hole (SBH) to Dynamic Cosmological Black Hole (CBH)}

Let us show now that $C B H$, with non-static $M M(13)$ is the cosmological limit of Schwarzschild's static metric (Stellar Black Hole, $S B H$ ). The latter is written (Outside the $S B H$ ):

$$
\mathrm{ds}^{2}=\left(1-\frac{R_{S}}{r}\right) \mathrm{d} r^{2}-\frac{1}{1-\frac{R_{S}}{r}} c^{2} \mathrm{~d} t^{2}, \quad r>R_{S} \quad \text { (Schwarz-out) }
$$

coupled with formula of Laplace ( $\left.R_{S}=\frac{2 G M_{S}}{c^{2}}\right)$. It is well known that the infinite behavior $r \mapsto \infty$ of (schwarz-out), brings back to the usual static Minkowskian 
limit $\mathrm{d} s^{2}=\mathrm{d} r^{2}-c^{2} \mathrm{~d} t^{2}$. A sad destiny for the $S B H$ which seems to be evaporated!

In dynamic NeoMinkowskian limit of $R G$ with $C C$ we have $S B H \underset{r \mapsto \infty}{\longrightarrow} C B H$ with $R_{S} \mapsto R_{H} \quad\left(M_{S} \mapsto M_{H}\right)$.

$$
\mathrm{d} s^{2}=\lim _{r \mapsto \infty}\left[\left(1-\frac{R_{S}}{r}\right) \mathrm{d} r^{2}-\frac{1}{1-\frac{R_{S}}{r}} c^{2} \mathrm{~d} t^{2}\right]=\mathrm{d} r^{2}-\frac{2 G M_{H}}{R_{H}} \mathrm{~d} t^{2} \quad \text { (Mink-out) (15-out) }
$$

This is a happy destiny for Stellar $S B H$ which becomes Universal $C B H(9)$.

It has been showed (Kruskal) that "singularity of Schwarzschild" $R_{S}$ is not a true physical singularity. We have to analyze the essential difference with the singularity (of Horizon) of Hubble $R_{H}$ which is a true physical (hyperbolic) singularity $(\S 3)$.

The structure of the paper will follow Feinberg's partition of NeoMinkowskian space time: Tachyon (OUT) $\S 1$, Photon (ON) $§ 2$, Bradyon (IN) $\S 3$ [6].

\section{2. [PHOTON] Emitted to the Inside: From CBH to CBR (Cosmological Background Radiation)}

NeoMinkowskian Universal coupling $\left(R_{H}, c\right)$ involves NECESSARILY a basic emission of waves (with constant velocity $c$ ) from the spherical surface of radius $R_{H} \quad$ (at singular initial time $t=0$ ).

Let us write LikeLight interval:

$$
\mathrm{d} s^{2}=\mathrm{d} r^{2}-\frac{2 G M_{H}}{R_{H}} \mathrm{~d} t^{2}=0 \Rightarrow \frac{\mathrm{d} r}{\mathrm{~d} t}=\sqrt{\frac{2 G M_{H}}{R_{H}}}=c \quad \text { (Mink-on) }
$$

(If there is no emission we have to apply the acceleration to the singular speed (of the photon) which becomes therefore no longer singular)

In what sense of radial direction should this radiation be emitted? To the outside or to the Inside of $C B H$ ?

Given that only tachyons can escape to the outside $(\S 1)$, the photons $(\S 2)$ can only be emitted TO THE INSIDE from the border $R_{H}$ and then in the bradyonic universe.

This is logically unstoppable.

Unless we invoke quantum fluctuations (Hawking 1974) in order to justify an emission of Black Radiation to the outside from event horizon of $S B H(\S 2-1)$.

Our basic emission of light resembles that of Hawking but it is not that of Hawking. Except on one point: the emitted radiation MUST be a Black Radiation. Indeed we have an emission of radiation from a global spherical surface of last diffusion, based on an isentropic transformation ( $2 \& 40)$.

Our $C B H$ is consistent with a singularity of the type Big Bang coupled with an emission of the type of $C B R$ (Cosmological Black (Background) Radiation (a $C B R$ at the Horizon of the $C B H$ ?). We will show in the next paragraph that is also compatible with an expansion of the type Hubble $\left(R_{H}\right.$ is an Hyperbolic Horizon, §3). 
We focus attention on a very important point that (Mink-on, 16) defines a NeoMinkowskian STRUCTURAL space-time velocity (von Ignatowski): it can correspond to light wave (photon) or gravific wave (graviton) as well (see Table 1).

\subsection{From Hawking's (Local) Black Radiation to (Global) CBR Black Radiation}

Let us remember that Hawking's black radiation [7]. is emitted from Horizon of Events of $S B H$ to the outside (see also Unruh, [8]). The expression "singularity of Schwarzschild" is henceforth outdated (still used in the old scientific literature). We confirm this point by making $\mathrm{ds}^{2}=0$ in Schwarzschild's metric (15):

$$
\left(1-\frac{R_{S}}{r}\right)^{2} \mathrm{~d} r^{2}-c^{2} \mathrm{~d} t^{2}=0, \quad \frac{\mathrm{d} r}{\mathrm{~d} t}=\lim _{r \mapsto R_{S}} \frac{c}{1-\frac{R_{S}}{r}} \mapsto \infty \text { (Schwarz-on) }
$$

We are indeed getting a non singular speed as large as we wish for $S B H$. But a speed of what? It is not speed of light $c$. Given that we are in the framework of $G R$, this supra-luminous speed can only be that of space point and therefore to the speed of propagation of gravitation (see Laplace, note 8).

In contrast with (Schwarz-on), for $C B H$ the singular Horizon of Hubble $R_{H}$ is coupled $\left(R_{H}, c\right)$ with singular Horizon of light velocity $c$ (Zero interval, or LightLike interval, see Table 1, we repeat 16-on, just above):

$$
\mathrm{d} r^{2}-\frac{2 G M_{H}}{R_{H}} \mathrm{~d} t^{2}=0, \quad \frac{\mathrm{d} r}{\mathrm{~d} t}=\sqrt{\frac{2 G M_{H}}{R_{H}}}=\dot{R}(0)=c, \quad r \mapsto R_{H} \quad \text { (Mink-on) (16-on) }
$$

Our Black Hole emits not only CBR (see Table 1 ) but also gravific waves at the speed of light (Poincaré, 1905, [4]) to the inside.

Inside the $S B H$ we have:

$$
\mathrm{d} s^{2}=c^{2} \frac{1}{\frac{R_{S}}{r}-1} \mathrm{~d} t^{2}-\left(\frac{R_{S}}{r}-1\right) \mathrm{d} r^{2}, \quad r<R_{S} \quad \text { (Schwarz-in) }
$$

\begin{tabular}{|c|c|}
\hline Schwarzschild's Horizon of $S B H \quad c^{2}=\frac{2 G M_{S}}{R_{S}}$ & Hubble's Horizon of $C B H \quad c^{2}=\frac{2 G M_{H}}{R_{H}}$ \\
\hline (Schwarz-out) & spacelike TACHYON (Mink-out) \\
\hline$r>R_{S} \quad(15)$ & $R(t)>R_{H}, \quad \dot{R}(t)>c, \quad \mathrm{~d} s^{2}=\mathrm{d} r^{2}-c^{2} \mathrm{~d} t^{2}$ \\
\hline Hawking's Black Radiation (outside) & lightlike PHOTON (Graviton?) (CBR inside) \\
\hline$r \mapsto R_{s}, \quad \frac{\mathrm{d} r}{\mathrm{~d} t} \mapsto \infty \quad$ (16) (Schwarz-on) & $R(0)=R_{H}, \quad \dot{R}(0)=c, \quad 0=\mathrm{d} r^{2}-c^{2} \mathrm{~d} t^{2} \quad($ Mink-on) \\
\hline & timelike BRADYON (Galaxy or Electron) \\
\hline$r<R_{S} \quad(17)($ Schwarz-in) & $r(t)<R_{H}, \quad \dot{r}(t)<c, \quad \mathrm{ds}^{2}=c^{2} \mathrm{~d} t^{2}-\mathrm{d} r^{2} \quad$ (Mink-in) \\
\hline
\end{tabular}

Table 1. Stellar black hole versus cosmological black hole. 
see Mink-in (17-in) in Table 1.

The density (1-1bis) is then internal density of $C B H$ (see $\S 3-3, \S 4)$ which can be written in cubic form of density of dark energy in $\frac{1}{R_{H}^{3}}$ and radiation form in $\frac{1}{R_{H}^{4}}$

$$
\rho_{\Lambda}=\frac{3 M_{H} c^{2}}{4 \pi R_{H}^{3}}=\frac{3 G}{2 \pi} \frac{M_{H}^{2}}{R_{H}^{4}}
$$

In the last case it's as if our universe $M_{H}$ is “in collision" with itself $M_{H}$.

\subsection{Tri-Partition of NeoMinkowskian Space-Time: TACHYON, PHOTON (GRAVITON) AND BRADYON (Table 1)}

We now present a summary (and also a plan) by transposing to our NeoMinkowskian World (spacelike, lightlike, timelike) the 3-partition of zones of usual $S B H r>R_{S}, r=R_{S}, r<R_{S}$ (see Table 1 first column, 15, 16, 17).

In second column of Table 1 we have 3-PARTITION according to velocity and according to space itself.

To each member of Feinberg's Trio (OUT, ON and IN) is attached a specific $M M$ (15-out, 16-on, 17-in).

We already have examined the tachyons $(\S 1)$ and (almost, $\S 2-3$ ) the photons $(\S 2)$. Finally we will examine non-baryonic bradyons galactic point $(\S 3)$ and electron $§ 4-5)$.

If it is almost impossible for an observer to get into a usual $S B H$, it is radically impossible for a (bradyonic) observer to get out of a $\mathrm{CBH}$.

Fortunately the interior of $C B H$ is precisely the Universe or the World itself. Fortunately also the observer is in the right place to measure the $C B R$ emission (see abstract).

This the reason why we suggest going beyond the notion the notion of "Hole" and replace it with that of the "W(hole) World" or "The Black Whole Universe" given that it is filled with Cosmological black radiation of the kind $C B R$ (emitted from Horizon of Hubble of $C B H$ (in $t=0$ ).

\subsection{Cosmological NeoMinkowskian Scale Hyperbolas and Perfect Cosmological Principle}

Given that cosmological kinematics is radial $(r=x)$ we can reduce space-time at $2 D$ usual diagram $(O t x)$ with scale unit Minkowskian Hyperbolas $x^{2}-c^{2} t^{2}=1$ and $c^{2} t^{2}-x^{2}=1$. Which is not usual in NeoMinkowskian space-time is that we have two singular Unit Hyperbolas directly induced by the $C C$.

Let us follow the photon that is emitted (towards the inside $(\S 3-2)$ in $t=0$ ) from $R_{H}$. It is received in $O$ at time $T_{H}$ (no negative time, see $\S 16$ ter).

Both points $\left(R_{H}, 0\right)$ and $\left(0, T_{H}\right)$ determine both singular Unit scale hyperbolas $2 D$ (more precisely half hyperbola in the first quadrant, respectively along axis $O x$ and along axis $O t)$ : 


$$
\begin{aligned}
x^{2}-c^{2} t^{2}=R_{H}^{2} \quad \text { (bradyonic) } & x=c t \quad \text { (photo-gravitonic) } \\
c^{2} t^{2}-x^{2}=T_{H}^{2} & \text { (tachyonic) }
\end{aligned}
$$

We rediscover the 3-partition with singular asymptote $(c=1)$ : a point on this asymptote can be photonic or gravitonic as well [4]. We can transform the coordinates of a point-graviton with $L T$ into another point-graviton on asymptote (with zero interval).

The first Hyperbole along $O_{X}$ (Tangent velocity WITHIN the light cone) is a bradyonic Hyperbole (see the Worldline ${ }^{10}$ of uniform acceleration §3) whilst the second Hyperbole, along $\mathrm{Ot}$, is a tachyonic Hyperbole (Tangent velocity OUT the light cone).

NeoMinkowskian hyperboles are compatible (a priori, see §3) with a Finite Time $\left(T_{H}=H_{\Lambda}^{-1}\right)$ underlying "BIG BANG" but also with a "PERFECT COSMOLOGICAL PRINCIPLE". Indeed we align a finite time $T_{H}$ on a finite space $R_{H}$ whilst in Steady State of Gold-Bondi-Hoyle, they align infinite time $T_{H}$ on a infinite space $R_{H}$. In our Hyperbolic Structure (Figure of Escher, see $\S 3)$, NeoMinkowskian space-time determine in fact a new kind of Steady-Sate.

We must now focus on bradyonic hyperbole (18. It must now be shown that Hyperbole (18) is the global (intergrated) form of local (differential) metric: $\mathrm{d} s^{2}=c^{2} \mathrm{~d} t^{2}-\mathrm{d} r^{2} \quad$ (Mink-in, see Table 1).

(The structure of the paper will follow Feinberg's 3-partition of NeoMinkowskian space time: Tachyon §1, Photon-Graviton §2, Bradyon §3)

\section{3. [BRADYON] Galaxies That Are Approaching a Hyperbolic Horizon (Escher Figures)}

Let the tachyons go (We caught the comet by the tail!) and install us in the inside of $C B H$ (in the Black Universe) in order to complete NeoMinkowskian pace-time with non-baryonic ( $\left.G_{\mu \nu}=0\right)$ bradyons ("as long as we have not everything, we have nothing"). The Whole Hole is the Whole Universe if we can express ourselves in this way.

\subsection{Boundary Conditions for Bradyons, Law of Hubble and Double Special Relativity (THESIS DSR)}

In order to introduce the second constant $R_{H}$ in $S R$ we have therefore to define a DSR (Doubly Special Relativity) with. Unlike standard DSR the second constant $R_{H}$ is not usual Planck's length but Hubble's length $R_{H}$ directly induced by $G R$ with $C C$ (if we cancel $C C(0)$ the second horizon disappears $R_{H}=R \rightarrow \infty$ ).

Everything happens as if the bradyons $r(t)<R_{H}-v(t)<c$ constitute a kind of inverted image (a kind of "Mirror Effect") of tachyons $R(t)>R_{H}-\dot{R}(t)>c$ with STRICT inequalities in both cases.

The Boundary Condition of coexistence of both Horizons $\left(c, R_{H}\right)$ (with standard notations for space points $v=u_{1}=v=c \beta$ ) is as follows:

${ }^{10}$ Minkowski himself (1908) considered that his theory was a cosmological theory of the Whole World (a trajectory becomes a WorldLine). 


$$
\frac{r(t)}{R_{H}}=\beta(t)=\frac{v(t)}{c}<1
$$

Inside the $C B H$ the (radial) law of Hubble, coupling "large velocity-large distance $(r=x)$ ", must therefore govern the expanding of galactic space points of the cosmological fluid (at a fixed time):

$$
v=c \beta=H_{\Lambda} r
$$

This CONDITION OF RADIALITY"11 (coupling "large velocity-inter-galactic distance") is quite basic. There is no expansion for "short" (non-large) distance and then for Planck's length.

The problem is now that it is difficult to see how a $M M$ (see Table 1 $\mathrm{d} s^{2}=c^{2} \mathrm{~d} t^{2}-\mathrm{d} r^{2}$, Mink-in) could be at the basis of such expanding kinematics (this is our THESIS).

Remember that so far there is only one solution (12) with spacelike signature $(-1,1,1,1)$ and that we are looking for a coupled solution with timelike signature $(+1,-1,-1,-1)$.

Thanks to the "mirror effect" we have a guide with the acceleration $\alpha_{M}$ (10, 11). Indeed if we have tachyonic minimal Horizon $R_{H}$ (coupled with maximal acceleration $\alpha_{M}$ ), we have now bradyonic minimal acceleration $\alpha_{M}$ (coupled with maximal Horizon $R_{H}$ ).

\subsection{Accelerated Motion of Material Point and Lobachevskian Velocity Space (HYPOTHESIS SR)}

Pauli remarks that the Worldline of usual (standard) relativistic Uniformly Accelerated Rectilinear Motion $U A R M$ in $S R$ coincide with usual scale hyperbola (along Ox, 18). Indeed we have the following hyperbolic worldline $U A R M$ (Minkowski 1908) in inertial system $K(t=0, v=0, \quad x=R)$ :

$$
r^{2}-c^{2} t^{2}=x^{2}-c^{2} t^{2}=R^{2}=\frac{c^{4}}{\alpha^{2}}, \frac{R \alpha}{c^{2}}=1
$$

In usual Minkowskian UARM $(R \rightarrow \infty, \alpha \rightarrow 0) \alpha$ represents a constant centrifugal (proper in $K^{\prime}$ ) acceleration $\alpha=\frac{\mathrm{d} \gamma(t) v(t)}{\mathrm{d} t}$ from which Minkowski deduced in 1908 by a double integration (18 bis). Minkowski's proper time of accelerated particle interests us particularly because it is directly connected with

$$
\begin{gathered}
c^{2} \mathrm{~d} t^{2}-\mathrm{d} x(t)^{2} \quad\left(v=\frac{\alpha t}{\sqrt{1+\frac{\alpha^{2} t^{2}}{c^{2}}}} \text { with } t=\tau=0\right): \\
\mathrm{d} \tau=\mathrm{d} t \sqrt{1-\frac{v^{2}(t)}{c^{2}}}=\mathrm{d} t \sqrt{1-\beta^{2}(t)}
\end{gathered}
$$

\footnotetext{
${ }^{11}$ This is the basic concept of Radial: Expansion only applies on a very large scale (distance and velocity). In fact this paper is devoted to translations (dark energy). The next will be devoted to rotation (dark matter, see conclusion). The one-third factor $\frac{1}{3}\left(c \sqrt{\frac{\Lambda}{3}}=H_{\Lambda}\right)$ are there to remind us that we are in space $3 D$ (see also initial ratio, 2 bis, electron and density-pressure for $E M$ radiation $\S 5$ ).
} 
Explicitly we have $\tau=\int_{0}^{t} \sqrt{1-\frac{v^{2}(t)}{c^{2}}} \mathrm{~d} t=\frac{c}{\alpha} \ln \left(\frac{\alpha}{c} t+\sqrt{1+\frac{\alpha^{2} t^{2}}{c^{2}}}\right)=\frac{c}{\alpha} \sinh ^{-1} \frac{\alpha t}{c}$. And then a hyperbolic sinus written with hyperbolic (index $h$ for hyperbolic) velocity $w_{h}=\frac{\alpha \tau}{c}{ }^{12}$ :

$$
\tau=\frac{c}{\alpha} \sinh ^{-1} \frac{\alpha t}{c} \Rightarrow t=\frac{c}{\alpha} \sinh \frac{\alpha \tau}{c}
$$

Using for hyperbola (anti-18) the hyperbolic polar coordinates $\left(R, w_{h}\right)$ (Varicak, Borel):

$$
\begin{gathered}
x=R \cosh w_{h}, \quad t=\frac{R}{C} \sinh w_{h} \\
\Rightarrow \quad \mathrm{d} x=R \mathrm{~d} w_{h} \sinh w_{h}, \quad \mathrm{~d} t=\frac{R}{C} \mathrm{~d} w_{h} \cosh w_{h}
\end{gathered}
$$

we deduce by differentiation (we refer 18 for Hyperboles, 19 for inequalities, 20 for metric):

$$
\mathrm{d} s^{2}=c^{2} \mathrm{~d} t^{2}-\mathrm{d} x(t)^{2}=R^{2} \mathrm{~d} w_{h}^{2}
$$

which corresponds to proper time (20) and "looks like" to Mink-in signature $(+1,-1,-1,-1)$.

We specify that $20-\mathrm{MM}$ is not a true $M M$ because either " $w_{h}$ is variable with fixed $R$ (one hyperbole)" or " $R$ is variable with fixed $w_{h}$ (a continuum set of hyperboles $x \leq R$ )".

This is the reason why Rindler () introduces a $N O N-M M$ for his cosmological model based on (UARM):

$$
\mathrm{d} s^{2}=R^{2} \mathrm{~d} w_{h}^{2}(t)-\mathrm{d} R(t)^{2}
$$

There is no singular hyperbole $R_{H}(18)^{13}(\mathrm{~d} R \neq 0)$.

Already in 1913, M. Born shows that the basic equations of UARM $x \geq R$ are also that of Rigid Motion $x \leq R$ of the axis $O x(O r)$ of Rigid motion of the "rod" $O R \quad x \leq R$ (a kind of Einstein's successive boost).

With Rigid Motion (a continuum set of hyperboles $x \leq R$ ) we are very close to concept of metric (see demonstration, §3-3).

Let us now return to the motion of accelerated material point and focus on his hyperbolic velocity which is defined by the inverse hyperbolic tangent (see 21)

$$
\begin{aligned}
& \frac{\mathrm{d} x(t)}{\mathrm{d} t}=\beta(t)=\tanh w_{h}(t) \Rightarrow w_{h}(t)=\tanh ^{-1} \beta(t) \\
& \Rightarrow \quad w_{h}(t)=\ln \sqrt{\frac{1+\beta(t)}{1-\beta(t)}}=\ln k_{h}(t)
\end{aligned}
$$

We have then a centrifugal proper acceleration which is derivative, with respect to proper time, of hyperbolic velocity:

\footnotetext{
${ }^{12}$ Or Rapidity of Robb: $w_{1}+w_{2}=w$.

${ }^{13}$ Rindler introduces, on the same basis (UARM) induces also a Black Hole but not a $C B H$.
} 


$$
\frac{c \mathrm{~d} w_{h}(t)}{\mathrm{d} \tau}=\alpha
$$

We have therefore here an accelerated Global (hyperbolic) motion which seems to correspond to (21-MM)? This kinematic UARM without dynamic (gravitation) is very strange (see Pauli, §3-3-3).

Let us now take a look to the geometry underlying UARM.

It is well known that in pseudoEuclidean $S R$, the space itself is Euclidean whilst the "space of velocity" is non-Euclidean, in Lobachevskian meaning (relativistic addition of velocities)

More precisely according to Borel [9] global "space of speed" is characterized by a negative curvature $-\frac{1}{c^{2}}$ given by the inverse of square of light velocity (velocity of light being the radius of curvature.

It is then useful to develop the geometrical aspect of the hypothesis. In Beltrami's disc model for Hyperbolic geometry we introduce Cayley-Klein's hyperbolic DISTANCE $d_{h}$ induced from the CROSS-RATIO formula is given by [10]:

$$
d_{h}=\tanh ^{-1} \frac{d}{d_{H}}
$$

where $d$ is usual distance defined smaller than $d<d_{H}$ the radius Horizon. If we apply this definition $\left(d_{h}, d\right.$ and $\left.d_{H}\right)$ to "space of velocity" ( $w_{h}, v$ and $c$ ) we rediscover (22):

$$
c w_{h}=c \tanh ^{-1} \beta=c \ln \sqrt{\frac{1+\beta}{1-\beta}}
$$

We underline that in usual $S R$ the velocity space is a true complete Hyperbolic space (with distance introducing by cross ratio) in strong sense of the term non-Euclidean (Penrose [11]).

This is not the case of the pseudo-hyperbolic spaces defined in Friedman's equation with $K=-1$ (where scale factor is given by a sinh (20) and not a tanh (24) which defines a Non-Euclidean Distance (24) (see §3-3-2) (Note in 21 bis that the time and the space are respectively given by a sinh and a cosh).

\subsection{DEMONSTRATION: From SR without Gravitation to Gravitational DSR}

The demonstration must be done in three stages (Gravitation and Geometry):

1) Hubble constant with its corresponding global Gravitation field (§3-3-1).

2) Law of Hubble itself with its corresponding global Geometry ( $\$ 3-3-2)$.

3) Finite Time from negative Lobatchevskian curvature: tanh versus sinh $(\S$ 3-3-3).

\subsubsection{NeoEinsteinian Principle of GLOBAL Equivalence between Gravitational Field and Minimal Acceleration}

Let us introduce now NeoMinkowskian singular bradyonic hyperbole (18) in $G R$ 
with $C C$ or, in other words, the second invariant (maximal) space $R_{H}$ (or minimal centrifugal acceleration $\alpha_{M}$ ) in $D S R$ :

$$
r^{2}-c^{2} t^{2}=x^{2}-c^{2} t^{2}=R_{H}^{2}=\frac{c^{4}}{\alpha_{M}^{2}}, \quad \frac{R_{H} \alpha_{M}}{c^{2}}=1
$$

Let's look closely at the limit (19) $r<R_{H}$. In truth we have first $R<R_{H}$. (anti-18, 18- $R_{H}$ ). Given that we have (Rindler 20, [12]) $r \leq R$ we deduce finally (19) $r<R_{H}$.

We have therefore now a true metric (Born's rigid motion becomes an expanding motion of space):

$$
\mathrm{d} s^{2}=c^{2} \mathrm{~d} t^{2}-\mathrm{d} x^{2}=R_{H}^{2} \mathrm{~d} w_{h}^{2}
$$

with hyperbolic coordinate $\left(R_{H}, w_{h}\right)$ on the basis $\left(\mathrm{d} R_{H}=0\right)$. In tensorial form $\eta_{\mu \nu}^{+}=\left(\begin{array}{cccc}1 & 0 & 0 & 0 \\ 0 & -1 & 0 & 0 \\ 0 & 0 & -1 & 0 \\ 0 & 0 & 0 & -1\end{array}\right)$ (20 G-MM).

This $M M\left(17\right.$-in) corresponds to a global gravitation field. ${ }^{14}(G-D S R)$ with Hubble Horizon:

$$
\frac{\mathrm{d} w_{h}(t)}{\mathrm{d} \tau}=H_{\Lambda}=\frac{\alpha_{M}}{c}=\frac{2 G M_{H}}{c R_{H}^{2}}
$$

The answer to first Pauli's question ("To which global gravitational field in GR does this UARM corresponds ${ }^{15}$ ? [13])" is then (23) and therefore (33). We answer with a global (cosmological) NeoEinsteinian principle of equivalence (23) between Milgrom's minimal centrifugal acceleration $\left(\alpha>\alpha_{M}\right)$ and expanding gravitation field within the $C B H$ (Universe).

Part one of the thesis ( $\$ 3-3-1)$ is thus demonstrated: we have Hubble constant but we do not yet have Hubble law itself (19bis) (§3-3-2). We are at this stage close (except Planck's mass) to cosmology of flat Universe [5].

\subsubsection{Induces in G-DSR a Global Negative Lobachevskian Curvature of Space (Escher)}

Until now our NeoMinkowskian Universe seems to be a Flat Universe, in the meaning of Tatum [5] because LOCAL Einsteinian curvature tensor is zero $\left(G_{\mu v}=0\right)$. There is no contradiction because we will prove now that there is a GLOBAL curvature for Euclidean 3D space (Penrose).

After the $G$ of Gravitation let us now deal the $G$ of Geometry. What about the geometry of space $3 D$ itself in $G$ - DSR?

Let us apply formula of Cayley-Klein's hyperbolic distance (24), respectively with $\left(s_{h}, r\right.$ and $\left.R_{H}\right)$, which can be considered here as the singular RADIUS OF

\footnotetext{
${ }^{14}$ We thus ended the forced cancellation of the gravitational NeoMinkowskian density (the putsch, note 1). We follow the same path as Unruh ([8]: Behind Hawking's local radiation there is a kinematic of uniform HYPERBOLIC acceleration. We transform therefore Unruh "local" effects into global (cosmological) effects (we change also the sense-and the meaning-of the emission).

${ }^{15}$ Historically Pauli did not find this field because he was using the $G R$ WITHOUT $C C$.
} 
ESCHER DISC (or Sphere):

$$
s_{h}=R_{H} \tanh ^{-1} \frac{r}{R_{H}}=R_{H} \ln \sqrt{\frac{1+\frac{r}{R_{H}}}{1-\frac{r}{R_{H}}}}=R_{H} \ln \sqrt{\frac{1+\beta}{1-\beta}}=R_{H} w_{h}
$$

The thesis, the (global) law of Hubble (19-19 bis)

$$
c \beta=c \frac{r}{R_{H}}=H_{\Lambda} r
$$

is then demonstrated (large distance-large velocity)! The GLOBAL (negative) curvature $\left(\frac{-1}{R_{H}^{2}}=-\frac{\Lambda}{3}\right)$ of the Whole Universe $(3 D)$ is then given by the $C C$ itself (this is the end for the putsch, note 1).

THIS IS AN EXTRAORDINARY RESULT: In $G$-DSR it is not only the "velocity space" (22 bis) but the "space itself" (25) that is Lobachevskian.

We obtain a whole (Global) Lobatchevskian "distance" (a scale factor) with hyperbolic function of tanh (22 and 24) and not a scale factor defined with sinh (21 bis and Friedman's model with $K=-1$ (see Penrose [11]). There is no contradiction between the cancellation of the local curvature factor $K=0$ in $R W$ metric (note 5).

Beltrami's abstract disc is concretized by famous aesthetic hyperbolic Escher's disc. The fact that the $\mathrm{W}$ (hole) Universe (with our observer) is inside a $C B H$ is not tragic because first we are enlightened by $C B R$ and then we fit in a harmonious (in Penrose's meaning) hyperbolic figure (disc) of Escher (Cayley-Klein's hyperbolic distance). Hyperbolic Universe is then compatible with radial expanding (without end) of galaxies and with Perfect Cosmological Principle (a horizon of finite space is aligned on a horizon of finite time).

While the tachyons move away as they escape (without end) from gravitational field (25) the galaxies move away infinitely (hyperbolic velocity) as they approach (without end) the (hyperbolic) horizon (§3-3-2) according to the law of Hubble (19-19 bis).

Milgrom's minimal acceleration [14] or Hubble's constant are then effects of hyperbolic (negative) curvature of space $3 D$.

By taking into account the $C C$ (Einstein 1917) in GR (Einstein 1915) and therefore a minimal acceleration $\alpha_{M}$ in equation of geodesic (Einstein 1915):

$$
\frac{\mathrm{d} u^{\mu}}{\mathrm{d} \tau}+\Gamma_{i j}^{\mu} u^{i} u^{j}=\frac{\mathrm{d} u^{\mu}}{\mathrm{d} \tau}+0 \neq 0 \Leftrightarrow \frac{\mathrm{d} u^{\mu}}{\mathrm{d} \tau} \neq 0
$$

Exactly as in the flat universe [5], Christoffel $\Gamma_{i j}^{\mu}$ cancel (with again disappearance of $u^{i} u^{j}$ (see $0-\mathrm{MM}$ ) but a radial component of acceleration does not cancel:

$$
\frac{\mathrm{d} u^{1}}{\mathrm{~d} \tau} \neq 0=\alpha_{M}=c^{2} \sqrt{\frac{\Lambda}{3}}
$$

So geodesics represent inertial systems with Straight lines "in global curved 
space" (or Hyperbolic Straight Lines). All our border relations are validated by hyperbolic global curvature (14, 14 bis, 7, 8, 12).

We have now not only a (Radial) Kinematic UARM (DSR based on the Duo $\left(c, R_{H}\right)$, but also a double (Scalar) Dynamic Newton's laws $(G-D S R)(8,13$ and 14):

$$
F_{G}=\frac{c^{4}}{2 G}=M_{H} \alpha_{M}, \quad f_{G}=m_{N B} \alpha_{M}
$$

Both are hyperbolic invariant but the second (see 14, §1-4) between a micromass $m_{N B}$ and macroacceleration $\alpha_{M}$ is enigmatic.

\subsubsection{Hyperbolic Tangent versus Hyperbolic Sinus:}

The "Big Bang", An Effect of (Negative) Curvature?

We have to apply the same hyperbolic definition (24) for space ( $s_{h}, r$ and $R_{H}$ ), and time $\left(\tau_{h}, t\right.$ and $\left.T_{H}\right)$ (contrast between sinh (21) and tanh (27) is obvious, see note 5$)$ :

$$
\tau_{h}=\tanh ^{-1} \frac{t}{T_{H}}=T_{H} \ln \sqrt{\frac{1+\frac{t}{T_{H}}}{1-\frac{t}{T_{H}}}}=T_{H} \ln \sqrt{\frac{1+\beta}{1-\beta}}=T_{H} w_{h}, \quad s_{h}=c \tau_{h}
$$

The comobile (proper) time (in $K)^{16} \tau_{h}$ in function of a parametric time $t$ (in $K)$. Parameter values of universal time in $K$ are included between 0 and $T_{H}$ $\left(0<t<T_{H}\right)$ in bradyonic Universe ("whole hole").

THIS IS AN EXTRAORDINARY RESULT!: The finite time $T_{H}$ ("Big Bang”) becomes an effect of the (negative) curvature of hyperbolic straight line of time (27).

Physical comobile time $\tau_{h}$ (also valid for comobile distance $s_{h}=c \tau_{h}$ ) is obviously INFINITE.

Since then the effective expanding universe (with infinite $s_{h}$ and infinite $\tau_{h}$ ) is a Steady State of Hoyle with (see §2-3) Perfect Cosmological Principle (with constant density and $C B R$ fluid defined at rest in $K$, see our deduction of the observed Temperature $\S 6)$.

The (double) derivative (10) with respect to the comobile time $\tau$ (represented here with a point):

$$
\dot{k}_{\text {Bradyons }}(t)=H_{\Lambda} k_{\text {Bradyons }}(t), \quad \ddot{k}(t)-H_{\Lambda}^{2} k(t)=0
$$

which is internal $E E$ corresponding to basic external $E E$ YP12 (4), (6-6 bis).

\footnotetext{
${ }^{16}$ Pauli was intrigued by the notion of proper acceleration in proper system $K^{\prime}$ which follows the fluid (medium) at rest in $K$ (in $S R$ ): In relativistic kinematics we will naturally describe by as "uniformly accelerated" a motion for which in a system $K^{\prime}$ moving with the medium or particle is always ot the same magnitude $\alpha$. The system $K^{\prime}$ is a different one at each instant; for one and the same Galilean system $K$ the acceleration of such a motion is not constant in time [13]. In $S R$ "The system $K^{\prime}$ is a different one at each instant" (successive $K$ - $K^{\prime}$ Lorentz boost). Pauli considers then a single global (hole) system $K^{\prime}$ is a non-Galilean system and moves therefore to $G R$ (without $C C$. which was not fashionable in his day, see note 16). According to Pauli, the proper time becomes then a COMOBILE time relative to (bradyonic) medium.
} 
( $\left.k=\mathrm{e}^{H_{\Lambda} \tau}, \dot{k}=H_{\Lambda} \mathrm{e}^{H_{\Lambda} \tau}\right)$. There is a "principle of correspondence" tachyons-bradyons (taking care of the correspondence of times):

$$
R(t) \leftrightarrow R_{H} k(t), \quad \dot{R}(t) \leftrightarrow R_{H} \dot{k}(t)=c k(t)
$$

The "fatal" objection of the note 3 is therefore refuted: Our basic equation $Y P 12(4)$ is perfectly relativistic (in $G R$ and in DSR meaning as well ${ }^{17}$ ).

In $G$-DSR Doppler Galactic Redshift formula $z=\frac{\Delta \lambda}{\lambda}=\frac{\lambda_{R}-\lambda_{E}}{\lambda_{E}}$ ) with fixed time (Reception and Emission), $t_{s}=t_{R}-t_{E}$ is given by hyperbolic factor $k_{h}$ which coincide with radial (note 10) or longitudinal Doppler factor of Bondi [15] $k_{B}$ :

$$
\begin{aligned}
k_{B} & =1+\mathrm{Z}=\frac{r\left(t_{R}\right)}{r\left(t_{E}\right)}=\frac{\lambda_{R}}{\lambda_{E}}=\mathrm{e}^{w_{h}}=\sqrt{\frac{1+\beta}{1-\beta}} \\
& \simeq 1+\beta+\frac{1}{2} \beta^{2}+\frac{1}{2} \beta^{3}+O\left(\beta^{4}\right) \\
& \simeq 1+H_{\Lambda} \frac{r}{c}+\frac{1}{2} H_{\Lambda}^{2} \frac{r^{2}}{c^{2}}+\cdots
\end{aligned}
$$

We notice a quadruple contrast.

In contrast with radial Doppler formula in $S R$ the velocity $\beta$ is the velocity of a space-point.

In contract with the other Redshift $\mathrm{z}$ formulas in $G R$, velocity $\beta$ is strictly infra-luminous $(<1)$.

In contrast with de Sitter, where there is not singular velocity for space-points) in $G$-DSR there is a double singular velocity for light wave (Photon) and for gravific wave (Graviton), as well.

In contrast with de Sitter, the expanding (29) $G$-DSR is only valid for large distances coupled with large velocities ${ }^{18}$.

\section{Intermediate Conclusions: Lepton Electron Is Not a Baryon}

Our NeoMinkowskian non-baryonic solution of $G R$ with $C C$ defines a Universe characterized by a (very large) finite Hyperbolic Hubble Horizon $R_{H}$ at its (singular) origin. Hyperbolic Universe is incompatible with inflation but compatible with the most undeniable observations: Galactic expansion and emission of non-galactic black radiation $(C B R)$ a (very long) finite time $R_{H}$ ago.

\footnotetext{
${ }^{17}$ Among the two speeds $\left(c \beta_{\text {bradyon }}, c k_{\text {tachyon }}\right)$ only the first is physical $(<c)$, the second $(>c)$ is a purely mathematical construction (a phase velocity). According to de Broglie ( $\$ 5-4)$, the supra-luminous velocity of electron (velocity of phase $\frac{c^{2}}{v}$ ) is coupled with its infra-luminous (velocity of group $v<c$ ) of electron. Can we replace the galactic point (Macro-force, 13) with an electron (micro-force $14)$ ?

${ }^{18}$ Both elongation of lengths and elongation of wavelengths are in the same boat exactly as in of Poincaré's Elongated Light Ellipsoids By applying the basic condition large velocity $v \approx c$-large distance $r \approx R_{H}$ they acquire a cosmic scope. According to Poincaré elongation (expansion) with a longitudinal factor $k$ is a consequence of Lorentz' contraction of factor $\gamma^{-1}$. Elongated Light Ellipsoids will be not developed here (see annex 2).
} 
Our paper could stop here.

The situation is somewhat disappointing because at this stage, we are not providing any new (numerical) information on the emission of the basic $C B R$.

The physicist must always be in search of unity: On one side we have NeoMinkowskian Vacuum with Gravitational Waves $\left(c, G, \Lambda, R_{H}, \alpha_{M}\right)$ whilst in the other side we have usual EM Minkowskian Vacuum (withOUT Gravitational Waves) with usual $E M$ constants (c, permittivity $\varepsilon$, permeability $\mu$, impedance $\Omega$, WITHOUT CHARGE $e$ ). Both sets seem irremediably disjoint.

The usual series of " $G c$ " $\frac{c^{2}}{2 G}, \frac{c^{4}}{2 G}, \frac{c^{5}}{2 G}$ respectively linear density, cosmological force $\left(F_{G}=\frac{c^{4}}{2 G}, 13\right)$, power of vacuum...(see L. Kostro) is not a real synthesis (only $c$ is in common, see 30 ).

Is there a missing element that is neither on one side (Macro) nor the other side (micro)?

ANSWER: the charge $e$. And therefore the concrete electron with charge $e$, mass $m_{e}$ and "classical" radius $r_{e}$ which is absent in $S R$ and (until now) in $G$-DSR as well.

According to Einstein's famous quotation: "electron $\left(e, m_{e}, r_{e}\right)$ is a stranger in classical electrodynamics"(Minkowskian Vacuum is without charge).

Until now NeoMinkowskian model is without non-baryonic light emitter.

Without the missing link, Poincaré's gravific electron (pressure of ether, §5), we cannot, at this stage make, any numerical evaluation or prediction.

\subsection{A (Stable) "Cosmological" Electron to Short Circuit the (Unstable) Primitive Atom?}

Let's test the candidate electron [16] in our equations $m_{N B}=m_{e} \quad(14,14$ bis):

$$
f_{G}=m_{e} \alpha_{M}=2 m_{e} G \frac{M_{H}}{R_{H}^{2}}
$$

This electro-dynamics (hyperbolic) force involves a connection between Macro $\left(M_{H}\right)$ and micromass $m_{e}$. It defines a "Cosmological (Global) Electron" which could answer to second question of Pauli (see first question, §3-3-1). "To which global EM field does ELECTRON in UARM corresponds?".

Pauli's answer ( $\mathrm{p}$ 93): "Hyperbolic motion thus constitutes a special case for which there is no formation of a wave zone nor any corresponding radiation". Pauli find a ZERO magnetic field (and then no radiation ${ }^{19}$ ) and hastens to specify that locally (parabolic) the electron emitted a radiation (see note 16 and also numerical annex).

In NeoMinkowskian limit of $G R$ with $C C$, we can suspect a link between this

\footnotetext{
${ }^{19}$ Einstein's Boost is based in $S R$ on infinitely slow acceleration ( $\alpha_{M} \rightarrow 0$ ), Einstein 1905 Pauli's deduction is logical because $U A R M$ consists in a series of infinitely slow boost globally without emission). In $G$-DSR we have a Big (cosmological) Boost with slow acceleration $\left(\alpha_{M}\right)$ globally with emission $(C B R)$. We remark that galactic Milgrom's minimal acceleration is here moved to cosmological radically relativistic ( $D S R$ and $G R$ ) framework.
} 
cosmological electron and cosmological radiation? The paper could then not stop here.

Note for this intermediate conclusions that Lorentz's electron has remained perfectly unperturbed (stable and elementary) for more than a century. Moreover Poincaré's discovers an enormous (negative) pressure ( $\$ 5-3)$ that ensures stability of the free electron in 1905.

Our future integrated (stable) electron is the ideal candidate to Short-Circuit (unstable) Planck's particle (Lemaître's primitive atom)?

Reset then by using following writing of $M M$ :

$$
\mathrm{ds}_{\text {tachyon }}^{2}=\frac{2 G M_{H}}{R_{H}} \mathrm{~d} t^{2}-\mathrm{d} r^{2}(10) \quad \Rightarrow \mathrm{d} s_{\text {electron }}^{2}=\frac{e^{2}}{m_{e} r_{e}} \mathrm{~d} t^{2}-\mathrm{d} r^{2}
$$

We could then proceed to an original electro-gravific synthesis under the presidency of $c$ (in other word a new non-usual approach of Einstein's unitary field):

$$
c^{2}=\frac{2 G M_{H}}{R_{H}}=\frac{e^{2}}{m_{e} r_{e}}
$$

\subsection{The First and the Second Density}

This enigmatic force $f_{G}$ of synthesis will have to be placed, next to $F_{G}=\frac{c^{4}}{2 G}=F_{\Lambda}$ (13) in the framework of usual electrostatic force $f_{e}$ coupled with (electro-)gravitational force $f_{G e}$ :

$$
F_{G}=\frac{c^{4}}{2 G} \text { (1) } \quad f_{e}=\frac{e^{2}}{r_{e}^{2}} \text { (2) } \quad f_{G e}=\frac{G m_{e}^{2}}{r_{e}^{2}}
$$

with the ratio of forces $\frac{f_{e}}{f_{G e}}=\frac{e^{2}}{G m_{e}^{2}}=\kappa_{G e} \approx 4.1604 \times 10^{42} \quad$ (see annex, numerical values Gauss-cgs) which becomes a basic universal constant. We have the following trio with:

$$
F_{G} \stackrel{\kappa_{G e}}{\longleftarrow} f_{e} \stackrel{\kappa_{G e}}{\longrightarrow} f_{G e}
$$

An electric force well framed by two gravific forces $\frac{2 F_{G}}{f_{e}}=\frac{f_{e}}{f_{G e}}=\kappa_{G e}$. Most physicists judge that the third force is ridiculous small compared to the other two (we note that $f_{G}$ is very close to $f_{G e}$ (see annex 1 )).

This balance of forces change completely if we consider the corresponding

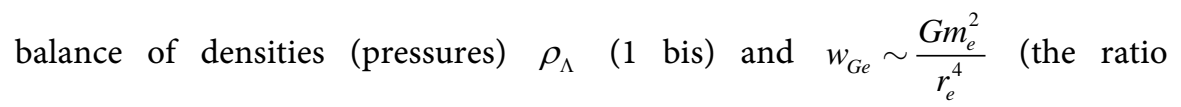
$\kappa_{G e}=\frac{w_{e}}{w_{G e}}=\frac{f_{e}}{f_{G e}}$ does not change)

$$
w_{e} \stackrel{\kappa_{G e}}{\longleftarrow} \rho_{\Lambda} \stackrel{\Omega}{\longleftarrow} w_{G e}
$$

The ratio $\frac{w_{G e}}{\rho_{\Lambda}}$ (see 12, the "unique" solution) being directly defined with 
$3\left(\frac{f_{G e}}{f_{G}}\right)^{2}$ of the order of $\Omega \simeq 5 \times 10^{-5} \quad$ (49 and 74, see numerical annex 1).

How can we justify the entry into scene of electron with this second density (pressure) in our NeoMinkowskian Universe?

At the beginning of $\S 3$ we claimed that "we caught the comet (devil) by the tail (tachyons)". We have now reached the head with bradyons:

$$
\begin{gathered}
T_{\mu \nu}^{\text {VACUUM }}=\rho_{\Lambda} \eta_{\mu \nu}^{-}=\rho_{\Lambda}\left(\begin{array}{cccc}
-1 & 0 & 0 & 0 \\
0 & 1 & 0 & 0 \\
0 & 0 & 1 & 0 \\
0 & 0 & 0 & 1
\end{array}\right) \text { (tachyons) } \\
\eta_{\mu \nu}^{+}=\left(\begin{array}{cccc}
1 & 0 & 0 & 0 \\
0 & -1 & 0 & 0 \\
0 & 0 & -1 & 0 \\
0 & 0 & 0 & -1
\end{array}\right) \text { (bradyons) }
\end{gathered}
$$

We draw attention to the fact that there is "tachyonic" FIRST density (dark energy) but until now no "bradyonic" (electro-photonic) SECOND density (see 57 bis).

There is no multiplier coefficient for timelike metric (Mink-in) unlike (12) for spacelike metric (1-1 bis, the Unique solution whatever the velocity). However for bradyons (and photons) inside the (W)Hole Universe we have another metric and why not another perfect fluid $E M$ tensor, another density, another pressure with $T_{\mu \nu}^{E M}=p_{e m} \eta_{\mu \nu}$ ?

In other words: would the metric (33-bradyons) be hidden in Perfect NeoMinkowskian fluid?

\section{The Second Density: From Electronic Density to Density of Gravific Waves}

Cosmologists distinguish three different types of Fluid which corresponds to three periods of the universe: 1$)$ the dust or inconsistent matter $(p=0), 2)$ the dark energy $p+\rho=0$ (anti-1) and 3) the so-called "Radiation" $p_{e m}=\frac{1}{3} w_{e m}$ (35) (generally reported, in cosmological literature to a "radiative period of Universe") $)^{20}$.

In cosmological literature the fluid (37) is always called "Fluid of Radiation" (always written in Riemannian metric $g_{\mu v}$ ).

$$
p_{e m}=\frac{1}{3} w_{e m} \Rightarrow T_{\mu v}^{E M}=\frac{4}{3} w_{e m} \frac{u_{\mu} u_{v}}{c^{2}}-\frac{1}{3} w_{e m} g_{\mu v}
$$

Nothing to do (at first sight) with electron.

\footnotetext{
${ }^{20}$ There are other fluids such as the ultra-relativistic electronic gas which seem not taken in consideration by cosmologists. So far no trace of the electron (charge $e$ and mass $m_{e}$ ) in cosmological usual representations.
} 


\subsection{Hidden Electron and Hidden Graviton in NeoMinkowskian Perfect Fluid "Radiation"}

We absolutely need to introduce the light (emission of $C B R$ in $t=0$ ) in NeoMinkowskian Fluid:

$$
T_{\mu \nu}^{E M}=\frac{4}{3} w_{e m} \frac{u_{\mu} u_{v}}{c^{2}}-\frac{1}{3} w_{e m} \eta_{\mu v}
$$

It is generally claimed that, if we replace $g_{\mu \nu}=\eta_{\mu \nu}$ in (34) from a Riemanian Fluid to a NeoMinkowskian Fluid the gravitation (and then gravific waves) would be eliminated (see the putsch note 1). It is obviously wrong because NeoMinkowskian limit imposes only a LIGHTLIKE 4-vector. The latter can correspond to a photon or could correspond to a (hypothetical) graviton (like light) as well.

In other words, for a classical light wave or a (hypothetical) "classical gravific wave" as well:

$$
\lambda_{P h}=\frac{\hbar}{P_{p h}}, v_{p h}=\frac{E}{\hbar} \quad\left(\lambda_{P h} v_{p h}=c\right) \quad \lambda_{G e}=\frac{a}{P_{G e}}, v_{G e}=\frac{E_{G e}}{a} \quad\left(\lambda_{G e} v_{G e}=c\right)
$$

(a being dimensionally an ACTION). We are therefore perfectly entitled to write next to "Radiation" Fluid (35) a "Gravitation" Fluid (36):

$$
T_{\mu \nu}^{G W}=\frac{4}{3} w_{G e} \frac{u_{\mu} u_{v}}{c^{2}}-\frac{1}{3} w_{G e} \eta_{\mu \nu} \Rightarrow p_{G e}=\frac{1}{3} w_{G e}
$$

where the index $G W$ refers to Poincarés Gravitational Waves and index Ge refers to Gravific-electron because there is a hidden electron in "Radiation" fluid of cosmologists $(\S 5-1)$.

Photon and Graviton are compatible with $G_{\mu v}=0$ (without rest mass) whilst Electron $p_{e}<\frac{1}{3} w_{e}$ (with leptonic rest mass) seems incompatible with $G_{\mu v}=0$.

The relationship (36) $T_{\mu v}^{G W}$ in $M M(33)$ is much more restrictive than it seemed at first glance.

We will prove that Poincaré's GW not only COULD exist but that they MUST exist (determined action $a$ and $\lambda_{G e}$ ).

The difficulty being here more logical than mathematical, we resume the situation with the following Table 2 (tachyons are gone):

Table 2. Photon, electron and graviton.

\begin{tabular}{ll}
\hline New trio ("GraPhoTron") & ON or IN singularity \\
\hline (spacelike) & $\left(T_{\mu v}=\rho \eta_{\mu v}, G_{\mu v}=0\right)$ \\
LIGHTLIKE (PHOTON or GRAVITON) & $p_{e m}=\frac{1}{3} w_{e n}, \quad p_{G e}=\frac{1}{3} w_{G e}$ \\
& $\left\|E_{P h}^{2}-P_{P h}^{2} c^{2}\right\|=0$ and Hypothetical $\left\|E_{G e}^{2}-P_{G e}^{2} c^{2}\right\|=0$ \\
timelike ELECTRON & $p_{e}<\frac{1}{3} w_{e},\left\|\gamma^{2} c^{2}-\gamma^{2} \beta^{2} c^{2}\right\|=c^{2}$ \\
\hline
\end{tabular}




\subsubsection{Hidden Electron in (Cosmological) Fluid "Radiation"}

For the first time we take now explicitly in consideration TIMELIKE 4-vectorial writing for velocities in the so called Radiation fluid (38).

Let us try to transpose the notations of the previous Table $2 \dot{R}(t), \dot{r}(t)$ using those of radial timelike 4 -vector velocity that can be reduced $\left(u_{0}, u_{1}\right)$ to temporal $u_{0}$ and spatial component $u_{1}$ (we stay at $3 D$ because we have $\left.w_{e m}=3 p_{e m}\right)$.

Given that it is impossible to put directly the velocity of light $c$ in such a timelike 4-vector, the radical singularity of our basic border " $v=c$ " is then structurally inscribed in temporal component $u_{0}=c$ for an "electronic point" (at rest) $u_{1}=0$.

There is then a hidden electron in Cosmological tensor of "Radiation" (a cosmological electron?). Let us see this crucial point in details:

$$
T_{\mu \nu}^{E M}=\left(\begin{array}{cccc}
w_{e m} & 0 & 0 & 0 \\
0 & p_{e m} & 0 & 0 \\
0 & 0 & p_{e m} & 0 \\
0 & 0 & 0 & p_{e m}
\end{array}\right)=\left(\begin{array}{cccc}
\frac{4}{3} w_{e m} & 0 & 0 & 0 \\
0 & 0 & 0 & 0 \\
0 & 0 & 0 & 0 \\
0 & 0 & 0 & 0
\end{array}\right)-p_{e m}\left(\begin{array}{cccc}
1 & 0 & 0 & 0 \\
0 & -1 & 0 & 0 \\
0 & 0 & -1 & 0 \\
0 & 0 & 0 & -1
\end{array}\right)
$$

1) The first tensor (left member) is usual $E M$ tensor of radiation with null trace $p_{e m}=\frac{1}{3} w_{e m}$. This is the reason why the perfect fluid (38) is called "Radiation" in cosmological literature.

2) The second tensor looks like that of an "electron" at rest $u_{1}=0$ which would hide behind its density (see Poincaré, §5-2).

3) The third tensor (of pressure) with timelike $M M$ is exactly the one we were looking (33) in Gravitational $G-D S R(\S 3-3,23-\mathrm{G}$ and 33$)$ with a (possible) way to the determination of the missing coefficient (or the second density).

This triple statement is not very original because it corresponds exactly to that of Poincaré in 1905 ("La dynamique de l'électron", §5-2). So far we have, at this stage an abstract electronic point but not yet a concrete electron $\left(e, m_{e}, r_{e}\right)$.

Let us remark that we can put in ( 35 or 37 ) the pressure to the left:

$$
T_{\mu v}^{E M}+p_{e m} \eta_{\mu v}=\frac{4}{3} w_{e m} \frac{u_{\mu} u_{v}}{c^{2}}
$$

In details:

$$
\left(\begin{array}{cccc}
w_{e m} & 0 & 0 & 0 \\
0 & \frac{1}{3} w_{e m} & 0 & 0 \\
0 & 0 & \frac{1}{3} w_{e m} & 0 \\
0 & 0 & 0 & \frac{1}{3} w_{e m}
\end{array}\right)+p_{e m}\left(\begin{array}{cccc}
1 & 0 & 0 & 0 \\
0 & -1 & 0 & 0 \\
0 & 0 & -1 & 0 \\
0 & 0 & 0 & -1
\end{array}\right)=\left(\begin{array}{cccc}
\frac{4}{3} w_{e m} & 0 & 0 & 0 \\
0 & 0 & 0 & 0 \\
0 & 0 & 0 & 0 \\
0 & 0 & 0 & 0
\end{array}\right) \text { (37 bis) }
$$

EM_RADIATION +?GRAVIFIC? (positive) PRESSURE- $\Rightarrow$ ?FREE ELECTRON?

Given that the putsch was canceled, we have also from (36) a hidden electron 
in Gravitation Field:

$$
T_{\mu v}^{G W}+p_{G e} \eta_{\mu v}=\frac{4}{3} w_{G e} \frac{u_{\mu} u_{v}}{c^{2}}
$$

This analysis shows that in "Radiation" tensor of Cosmologists there is not only a hidden electron but also a connection of this electron with gravific waves.

\subsubsection{Hidden Electron in (Cosmological) Black Radiation}

The concrete radiation in our cosmological problematic is black radiation in $C B R$. Let us remark, in this respect, that the situation of concrete electron $\left(e, m_{e}, r_{e}\right)$ is exactly the same. All formulas of Planck's black body are with Planck's constant $h$ and without $\left(e, m_{e}, r_{e}\right)$.

The concrete electron $\left(e, m_{e}, r_{e}\right)$ is hidden (behind or below $h$ ) while the black radiation is emitted by electronic oscillators ${ }^{21}$.

Everything happens as if concrete electron is excluded both in the fluid and in the black body (for example in the formula of Stephan-Boltzmann, see $§ 6$ and annex 1).

Let us finally note that according cosmological usual "Isentropic Expansion of Spherical CBR" (see basic Equation (1)-anti(1)):

$$
\begin{aligned}
& \mathrm{d} U+\frac{1}{3} w_{e m} \mathrm{~d} V=T \mathrm{~d} S=0 \\
& \mathrm{~d}\left(w_{e m} \frac{4}{3} \pi R^{3}\right)+\frac{1}{3} w_{e m} \mathrm{~d}\left(\frac{4}{3} \pi R^{3}\right)=0
\end{aligned}
$$

the variable density $w_{e m}$ depends on variable Radius (in $\frac{1}{R^{4}}$ ) connected with variable Temperature $T$ (in degrees Kelvin K) with formula of Stephan-Boltzmann (see annex 1):

$$
w_{e m}=\sigma_{\text {Stephan }} T^{4}
$$

(see annex 1,80$)$

$$
w_{e m} \sim \frac{1}{R^{4}} \Rightarrow T^{4} \sim \frac{1}{R^{4}} \Rightarrow R T=\text { cte }
$$

Remember that we have a fixed hyperbolic horizon $R_{H}$ (directly induced by $C C, \rho_{\Lambda}=\frac{3 G}{2 \pi} \frac{M_{H}^{2}}{R_{H}^{4}}$ ) which could correspond to a new basic constant Temperature $T_{K}$ of our Black Universe ( $(6)$ do not confuse with Time of Hubble $T_{H}$ ). Everything happens as if Radiation Fluid and Black Radiation were the two sides of the same coin (electronic).

\subsection{Synthesis between Poincaré's Gravific Electron and Poincaré's Gravific Waves}

We have to find a new synthesis for our Trio: ELECTRON-PHOTON

${ }^{21}$ Given that non-baryonic ( $G_{\mu v}=0$ ) Redshift is based on light emission by galactic... baryons, we are missing non-baryonic emitters. Leptonic electron (a renowned emitter!) could be a good candidate $\S 3-6$ and $\S 4)$. 
(L-Wave)-GRAVITON (G-Wave). In order to do that we need to start a concrete electron $\left(e, m_{e}, r_{e}\right)$.

\subsubsection{Poincaré's Historical Induction of the Mass of Electron with Gravific Pressure}

In 1905 in his basic paper on "La Dynamique de l'Electron, Poincaré is looking for a determination of mass $m_{e}$ electron from its $E M$ emitted field (July, $\S 6$ Lorentz' Contraction, see note 20). He discovers that a purely $E M$ induction of the mass is not possible because we have to take into account a strange Non-EM pressure (probably) of gravitational origin [4].

From Energy-Impusion tensor $T_{\mu \nu}^{E M}$ Poincaré notes (with $L T$ ) that energy and impulsion of a purely $E M$ Electron are not transformed $\left(E_{0}=m c^{2}\right)$ as the components of a timelike 4-vector: it appears parasitic factors 1/3, 4/3:

$$
E_{e}=\gamma\left(1+\frac{1}{3} \beta^{2}\right) E_{0}, \quad P_{e}=\frac{4}{3} \gamma \beta E_{0} \stackrel{\text { Gravific Pressure }}{\longrightarrow} E_{e}=\gamma E_{0}, \quad P=\gamma \beta E_{0}
$$

Poincare then adds to the $E M$ tensor a Non-EM tensor in such a way that these parasitic thirds are eliminated (41). Mathematically it means that the diagonal terms of new tensor $\left(T_{\mu v}^{E M}+T_{\mu v}^{N o n-E M}\right)$ are compensated, except the first one (00) $w_{e m}=\frac{1}{8 \pi} E_{l}^{2}=\frac{1}{8 \pi}\left(\frac{e}{r^{2}}\right)^{2}$ (Electric field $E_{l}$ ) in the system of electron at rest $\left(w_{e m}=\frac{E_{l}^{2}+H^{2}}{8 \pi}\right)$ with $H=0$, see 45 and Landau 31-5, p106, [17]).

Usually 4-tensor Energy-Impulsion can be reduced to a 4-vector Energy-Impulsion only in the absence of a charge (Minkowskian Vacuum is without charge, see introduction). Thanks to Poincaré's Gravific Pressure, 4-tensor Energy-Impulsion can be reduced to a 4-vector Energy-Impulsion also in presence of a (spherical) charge $e$ (41). The basis idea is to define the electron $\left(w_{e}\right)$ from its field $\left(w_{e m}\right)$ :

$$
w_{e}=w_{e m}
$$

This internal density $w_{e}(40)$ in the electron (according to Poincaré) is explicitly written by Langevin (in 1913) on the basis of the model of surface charge distribution in the spherical radius $r_{e}$ of Poincaré's electron or "hole in ether"22 (in details we have after integration $\frac{4}{3} \pi r_{e}^{3} w_{e}=\frac{1}{3} \frac{e^{2}}{2 r_{e}}$ ) (1913):

$$
w_{e}=\frac{1}{8 \pi} \frac{e^{2}}{r_{e}^{4}}
$$

Poincaré does not write in 1905 any formula for its internal density (or pressure) but specifies (in the sentence where he claims gravitational origin) that the density is proportional to the "fourth power of experimental mass $m_{e}$ of elec-

\footnotetext{
${ }^{22}$ Such a name "classical radius of electron" is inappropriate in Poincaré's theory because he designates his electron as a "Hole in the ether". In modern language this is similar to that of quantum theory of field (QED of Dirac-Feynman): Poincaré's electron would be "singularity in the field". From which cosmological field the mass of electron is inducted? We are waiting for the answer from QED. We have a WED answer (\$5-6).
} 
tron". With basic relation (32 bis) we find indeed the proportionality with $m_{e}^{4}$ announced by Poincaré:

$$
r=\frac{e^{2}}{m_{e} c^{2}}=r_{e} \Rightarrow w_{e}=\frac{1}{8 \pi} \frac{m_{e}^{4} c^{8}}{e^{6}}=\frac{1}{8 \pi} \frac{m_{e}}{r_{e}^{3}} \Rightarrow m_{e}=8 \pi w_{e} r_{e}^{3}
$$

The mass $m_{e}$ of free electron is therefore entirely induced from cubic (in volume) density of mass $w_{e}$ by taking into account the surface distribution of the charge. This classical attempt to integrate the concrete electron $\left(r_{e}, e, m_{e}\right)$ in the framework of $S R$ was historically not followed because such a gravific ether is obviously unthinkable from the dominant Einsteinian point of view (1905, June, removal of ether). The few physicists who became interested (Langevin, von Laue, Born, Fermi) in Poincaré's supplementary Potential (overthrowed by qnantum theory), interpreted as a purely $\mathrm{AD}$ HOC anti-electrostatic pressure without any (Wave) ElectroDynamics perspective underlying Poincarés continuous approach.

According to Poincaré the main physical argument for the gravitational origin of his pressure is that it must be attractive (anti-electrostatic). With the same argument being that, in a rather enigmatic way, that his gravific pressure must be negative (nothing to do here with $p+\rho=0$ ).

In scientific literature about Poincaré's pressure (Langevin, von Laue, Fermi ...) the $E M$ factor $1 / 3$ between $w_{e m}$ and $p_{e m}$ is obviously NOT extended (except Born see below) between gravific $p_{e}$ and gravific $w_{e}$.

\subsubsection{Poincaré's Negative Pressure and NeoMinkowskian Perfect Fluid} Poincaré's historical deduction ( $\$ 6$ Lorentz contraction, [4]) has apparently nothing to do with equation of Perfect fluid (formulated apparently by von Laue or Born ten years after 1905). And therefore with (35 bis-36 bis). Let us try to extract, with the perfect fluid, the deepest root of Poincare reasoning: the role of NEGATIVE pressure in the addition of two basic tensors $(E M+N o n-E M)$ (we do a reconstitution as in a judicial investigation).

Poincaré wonders: "Which tensor should I add to the first in order to remove the diagonal terms in the third"? (except the first one (00), 42):

$$
\left(\begin{array}{cccc}
w_{e m} & 0 & 0 & 0 \\
0 & \frac{1}{3} w_{e m} & 0 & 0 \\
0 & 0 & \frac{1}{3} w_{e m} & 0 \\
0 & 0 & 0 & \frac{1}{3} w_{e m}
\end{array}\right)+\left(\begin{array}{cccc}
? w_{e m} & 0 & 0 & 0 \\
0 & p_{e m} ? & 0 & 0 \\
0 & 0 & p_{e m} ? & 0 \\
0 & 0 & 0 & p_{e m} ?
\end{array}\right)=\left(\begin{array}{cccc}
? w_{e m} & 0 & 0 & 0 \\
0 & 0 & 0 & 0 \\
0 & 0 & 0 & 0 \\
0 & 0 & 0 & 0
\end{array}\right)
$$

M (The first is classical $E M$ with zero trace). Poincaré's mathematical answer would be logically:

$$
\left(\begin{array}{cccc}
\frac{1}{3} w_{e m} & 0 & 0 & 0 \\
0 & p_{e m}=-\frac{1}{3} w_{e m} & 0 & 0 \\
0 & 0 & p_{e m}=-\frac{1}{3} w_{e m} & 0 \\
0 & 0 & 0 & p_{e m}=-\frac{1}{3} w_{e m}
\end{array}\right)
$$


and therefore mathematically the gravific pressure indeed must be negative $p=-\frac{1}{3} w_{e}$ because it is then NOT an $E M$ pressure $p=+\frac{1}{3} w_{e}$ (non zero trace $\left.\frac{4}{3} w_{e}\right)$. Physically his gravific pressure is anti-electrostatic repulsion.

Let us develop the addition $(E M+N o n-E M)$ (with 45):

$$
\begin{aligned}
& \left(\begin{array}{cccc}
w_{e m} & 0 & 0 & 0 \\
0 & \frac{1}{3} w_{e m} & 0 & 0 \\
0 & 0 & \frac{1}{3} w_{e m} & 0 \\
0 & 0 & 0 & \frac{1}{3} w_{e m}
\end{array}\right)+\left(\begin{array}{cccc}
\frac{1}{3} w_{e m} & 0 & 0 & 0 \\
0 & -\frac{1}{3} w_{e m} & 0 & 0 \\
0 & 0 & -\frac{1}{3} w_{e m} & 0 \\
0 & 0 & 0 & -\frac{1}{3} w_{e m}
\end{array}\right) \\
& =\left(\begin{array}{cccc}
\frac{4}{3} w_{e m} & 0 & 0 & 0 \\
0 & 0 & 0 & 0 \\
0 & 0 & 0 & 0 \\
0 & 0 & 0 & 0
\end{array}\right)
\end{aligned}
$$

This is exactly the perfect fluid (39) with an electron at rest (§5-1).

EM_RADIATION + GRAVIFIC NEGATIVE PRESSURE- $\Rightarrow$ FREE ELECTRON?

(see 37 bis) Therefore Poincaré's historical (long) deduction is the same as our deduction $g_{\mu v}=\eta_{\mu v}$ from Riemanian Fluid to NeoMinkowskian Fluid (35-36-37).

There is however a CRUCIAL CONTRAST because (35-36-37) is formulated with $M M$ :

$$
p_{e} \eta_{\mu v}=\frac{1}{3} \frac{1}{8 \pi} \frac{e^{2}}{r_{e}^{4}}\left(\begin{array}{cccc}
1 & 0 & 0 & 0 \\
0 & -1 & 0 & 0 \\
0 & 0 & -1 & 0 \\
0 & 0 & 0 & -1
\end{array}\right)
$$

and therefore the pressure is no longer negative!

Poincaré's negative gravific pressure becomes in NeoMinkowskian Fluid a positive pressure with $M M$ (see 37 bis and 37 ter).

Nothing prevents then to affirm (dixit Born) that everything returns in a purely EMorder with $p_{e}=+\frac{1}{3} w_{e}$ aligned on radiation $p_{e m}=\frac{1}{3} w_{e m}$ !

Equation (35-36-37) exerts a huge constraint that did not exist in Poincaré's historical calculation. The gravitational origin of Poincaré's pressure seems to have evaporated but the basic relationship of purely EM fluid (Born) is contradictory because we should have $p_{e}<\frac{1}{3} w_{e}$ for any electron (see $\S 5-2-4$ ).

The situation seems hopeless so we must ask questions (we propose the three following questions). 


\subsubsection{Did Poincaré (Langevin) Choose the Right Density?}

Did Poincaré (Langevin) choose the right density? In other words: Is the coefficient (density $w_{e}$ ) considered the right one in (33)?

The missing link (see 33) density of the anti-electrostatic force is very huge $10^{8}$ $\mathrm{g} / \mathrm{cm}^{3}$ (stability of elementar electron) and not very credible in the role of density of radiation, Reported to black radiation of $C B R$ this first attempt involves $w_{e}=w_{C B R}$ (see 40 , the density involves a temperature) we obtain about $10^{15} \mathrm{~K}$ !

At this stage we have not discovered yet the missing coefficient.

Moreover Langevin-Poincaré's density (35-10) is connected with gravitational theory but the constant $G$ is hidden (in 48).

Constant $G$ is however NOT hidden in the third formula of gravific density.

Remember $(\S 4)$ that there are three densities that we can report (logically) to Feinberg's trio (tachyon, electron, photon (+graviton):

$$
\begin{gathered}
\rho_{\Lambda}=\rho=\frac{\Lambda c^{4}}{8 \pi G}(1) \\
w_{e}=\frac{1}{8 \pi} \frac{e^{2}}{r_{e}^{4}}(2) \\
w_{G e}=\frac{G}{8 \pi} \frac{m_{e}^{2}}{r_{e}^{4}}=\frac{w_{e}}{\kappa}=w_{P h o t o n}
\end{gathered}
$$

1) The first tachyonic density is the density of dark Energy ( $C C)$.

2) The second density is internal mass density of electron (Poincaré's formula, 44).

3) The third enigmatic ( $G e$ ) Gravifico-electronic (very tiny) density (40) with very weak gravitational long range force (in contrast with the first, huge gravitational long range force).

The ratio $\kappa_{G e}=\frac{w_{e}}{w_{G e}}=\frac{f_{e}}{f_{G e}}$ does not change.

This third density could be reported (logically) to Photon or Graviton, Radiation or Gravitation. The question is: a density of what? (this is not a density of mass in electron, see 44).

It can only be a density of light waves or density of gravific waves.

\subsubsection{Can an Electron Moving at the Speed of Light Turn into ... Photon?}

Let us go back to Born's deadlock. Such an alignment of electron $p_{e}=\frac{1}{3} w_{e}$ on photon $p_{e m}=\frac{1}{3} w_{e m}$ is impossible. Indeed if we follow the equation of fluid of radiation (35-37-38) (this is not the case of historical Poincaré's demonstration) it is a real problem because we have now, from the symmetry underlying NeoMinkowskian fluid, $p_{e}=\frac{1}{3} w_{e}$ while we should have $p_{e}<\frac{1}{3} w_{e}$ !

In Summary, with underlying symmetry in "radiation" fluid (35) that: involves " $p=\frac{1}{3} w$ " for photon and for electron as well. Consequently we have 
" $E=P c$ " for electron (with non zero proper mass) and photon (with zero proper mass) as well! In other words, can an electron moving at the speed of light turn into ... photon? No! Ask the (almost) same question but otherwise.

\subsubsection{Does the "Perfect Ultra-Relativistic Electron" ("PURE") Exist?}

For photonic gas we have rigorous relationship $p_{p h}=\frac{1}{3} w_{p h}$ whilst for an Ultra-Relativistic (hot) electronic gas we have an approximate relationship $p_{e} \approx \frac{1}{3} w_{e}$. "Ultra-relativistic" usually means that the proper energy (mass at rest) $E_{0}=m_{0} c^{2}$ of the electron becomes negligible (almost zero) compared to its kinetic energy $\gamma \gg 1$ ).

Perfect Ultra-Relativistic (" $v=c$ ”) Electron (PURE) with zero mass seems impossible because leptonic electron has a (proper) mass. The situation seems desperate because (35) leads inexorably to a contradiction between $p_{e} \approx \frac{1}{3} w_{e}$ and the limit $p_{e}=\frac{1}{3} w_{e}$.

That is to say: Does the "PURE" exist?

If limit electron $(v=c)$ or Cosmological Electron does not exist, then the primitive atom exists.

All the above is based on ( 35 and 35 bis $)^{23}$. So let's forget for a few moments (35) and let's take a look on (36 and 36 bis).

Remember that Poincaré never establishes any direct link between his GRAVIFIC waves (introduction) and his GRAVIFIC pressure on electron (§6) $[4])$.

\subsection{From ELECTRON TO GRAVITON: Synthesis towards (Second) Density of Gravific Waves}

Let's reverse the reasoning from (36) that defines the Graviton (or Gravific Wave) by assuming that there is (maybe) a link with "PUR" electron $(G e)$.

If PURE exists then it defines a Gravific wave (lightlike Graviton, SEE previous Table 2 and [16]):

$$
\left\|E_{G e}^{2}-P_{G e}^{2} c^{2}\right\|=0, \quad \lambda_{G e}=\frac{a}{P_{G e}}, \quad v_{G}=\frac{E_{G e}}{a} \quad\left(\lambda_{G e} v_{G e}=c\right)
$$

where $\left(P_{G e}, E_{G e}\right)$ are respectively impulsion and energy of "light" electron (the graviton) where the "Action" a remains to be determined (52).

Let's locate the problem at the level of basic relation (in NeoMinkowskian approach) for electron ${ }^{24}$ :

\footnotetext{
${ }^{23}$ Such a passage (from 35) to the limit, $\left.\beta \rightarrow 1(v \rightarrow c), m_{0} \rightarrow 0, \gamma \rightarrow \infty\right)$ from a timelike 4-vector $\left\|\gamma^{2} m_{0}^{2} c^{4}-\gamma^{2} m_{0}^{2} \beta^{2} c^{4}\right\|=E_{0}^{2}=\left(m_{0} c^{2}\right)^{2}$ to a lightlike 4-vector is obviously impossible (indeterminate (35). This PUR or light Electron $(v=c)$ is an Horror (exactly as Einstein's LichtKomplex (1905) is a terror (see annex 2, historical epilogue).

${ }^{24}$ It doesn't exist such a relationship for baryons. Evidence: the quarks.
} 


$$
r_{e}=\frac{e^{2} / c}{m_{e} c}=\frac{e^{2} / c}{P_{G e}} \Rightarrow \frac{r_{e}}{c}=\frac{e^{2} / c}{m_{e} c^{2}}=\frac{e^{2} / c}{E_{G e}}
$$

The first $P_{G e}=m_{e} c$ is an Horror (a "PURE" Horror) whilst the second $E_{G e}=m_{e} c^{2}$ is a Wonder (curious discrimination!).

Given that Poincaré's concept of "Hole in ether" recalls de Broglie's Diffraction of an electronic wave in "hole of a screen", let us associate to Poincaré's length $r_{e}$ a de Broglie's wave $\left(\lambda_{G e}, v_{G e}\right)$ [16]:

$$
\lambda_{G e}=\frac{e^{2} / c}{P_{G e}}=\frac{e^{2} / c}{m_{e} c} \rightleftharpoons v_{G e}=\frac{E_{G e}}{e^{2} / c}=\frac{m_{e} c^{2}}{e^{2} / c}
$$

that defines logically a wavelength and then a frequency and finally a "lightlike gravitational wave" $\lambda_{G e} v_{G e}=c$ or a lightlike graviton $E_{G e}=P_{G e} c$ (a radius is a length that becomes a wavelength and therefore a wavelength)

$$
P_{G e}=\frac{e^{2} / c}{\lambda_{G e}} \rightleftharpoons E_{G e}=\left(e^{2} / c\right) v_{G e}
$$

where Impulsion $P_{G e}$ Energy $E_{G e}$ with Action $a$ is now determined $\left(a=e^{2} / c\right.$ replaces $\hbar)$. CQFD.

Does the (Cosmological) "PURE" exist? The answer is: YES but it's not the photon: it is the GRAVITON.

The wavelength associated with the Graviton $\lambda_{G e}$ is not the wavelength $\lambda_{e}$ (Compton) of a (quantum) electron:

$$
P_{e}=\frac{\hbar}{\lambda_{e}} \rightleftharpoons E_{e}=\hbar v_{e}
$$

A GRAVITON is A PUR (WAVE $v=c$ ) ELECTRON WHOSE THE WAVELENGTH IS $\lambda_{G e}$.

Frequency $v_{G e}$ will be connected with angular velocity of Thomas $\omega_{T}=2 \pi v_{G e}$ see conclusions ${ }^{25}$.

The limit electron does not give a photon but a graviton ${ }^{26}$.

Concretely it means that gravific waves (1905, intro) are inseparable from a very special gravific density connected to Poincaré's electron $(1905, \S 6)$.

The lightlike graviton is without proper mass. The mass of electron is in fact carried by the $G$-wave in (51). We suggest calling this mass $m_{e}$ (without proper mass $G_{\mu v}=0$ ) a "comobile mass" of graviton (without charge ${ }^{27}$ ) exactly like galactic fluid (see also comobile time, §3-5).

In NeoMinkowskian $G$-DSR the "Wonder" $E_{G e}=m_{e} c^{2}$ and the "Horror" $P_{G e}=m_{e} c$ will be on the same boat (end of the discrimination, 50).

Let us specify also the ratio with wavelength of Compton and radius of Bohr (with fine structure constant):

\footnotetext{
${ }^{25}$ See proportionality Energy-Frequency of LichtKomplex of the young Einstein in annex 2.

${ }^{26}$ Given that RADIAL Kinematics corresponds in this paper, to SCALAR Dynamics, Graviton might have something to do with SCALAR Boson (Englert, ULB).

${ }^{27}$ Charge disappears in a pure coefficient of proportionality $e^{2} / c$ (see $\left.\$ 5-4\right)$.
} 


$$
\frac{\lambda_{e}}{\lambda_{G e}}=\frac{\hbar}{e^{2} / c}, \quad \frac{\lambda_{\text {Bohr }}}{\lambda_{G e}}=\left(\frac{\lambda_{e}}{\lambda_{G e}}\right)^{2}=\left(\frac{\hbar c}{e^{2}}\right)^{2}
$$

(see 59, ratio of densities).

Objection: This is a Graviton without Gravity (without constant $G$ )?

Answer: $G$ is in the amplitude (energetic intensity) of the $G$-wave. Gravity $(G)$ is directly inscribed in density of energy of $G$-interaction between two point electron (a "double electron" $m_{e} m_{e}$, see 16-on) separated by basic wavelength $\lambda_{G e}$ (a kind of "string"):

$$
f_{G e}=G \frac{m_{e}^{2}}{\lambda_{G e}^{2}}(30-3) \quad w_{G e}=\frac{G}{8 \pi} \frac{m_{e}^{2}}{\lambda_{G e}^{4}} \quad(49-3)
$$

So (49-3) is a density of Poincaré's Gravific Waves. Most physicists think that the gravitational (density of) force between electrons (separated with $\lambda_{G e}$ ) is perfectly negligible. This verdict was true before 1965 and it's still true after 1965.

Poincaré had the right reasoning $(\S 5-2)$ but not the right density (see $49-3$, in 54 and $\S 6)$.

\subsection{GRAVIFIC SUBQUANTUM SUBSTRATUM: WED (Poincaré-de Broglie) versus QED (Dirac-Feynman)}

de Broglie, specialist of the diffraction of the wave electron, distinguishes (in 1957) three basic levels in physics ${ }^{28}$.

1) The first level is (macroscopic) according to de Broglie is classical physics (dynamic and thermodynamics).

2) The second level is (microscopic) Quantum physics (baryonic or atomic matter $\left.G_{\mu v} \neq 0\right)$.

3) The third level (hypomicroscopic) is the deepest level (photonic-electronic, non baryonic $G_{\mu v}=0$ ): "the deepest level is Hypomicrophysics SubQuantum Substratum constituted by this Vacuum a huge reservoir of underlying energy of which we still know almost nothing" (in French: Le niveau le plus profond, hypomicrophysique ou subquantique pourrait-on dire, constitu? par ce "vide" réservoir immense dénergie sous-jacente dont nous ignorons encore presque tout)

The third level "Hypomicrophysics SubQuantum Substratum" is particularly suitable for our problematic.

According to de Broglie, relativistic Wave Mechanics or Wave ElectroDy-

${ }^{28}$ Cours professé par de Broglie à la Sorbonne durant l'année scolaire 1957-1958 [16]:

1) le niveau macrophysique des phénomènes macroscopiques directement observables? notre échelle qui est le domaine propre de la Physique dite classique (the macrophysic level of macroscopic phenomena directly observable on our scale which is the proper domain of so-called classical physics).

2) le niveau microphysique ou quantique qui est celui des molécules, des atomes, des noyaux ou plus généralement des particules élémentaires, qui est le domaine propre de la Physique quantique. (the microphysical or quantum level which is that of molecules, atoms, nuclei or more generally elementary particles, which is the proper domain of quantum physics):

The first level is (macroscopic) according to de Broglie is classical physics. 
namic ( WED) (§5) should preside over the destiny of Quantum Mechanics or Quantum ElectroDynamic $(Q E D)$.

We know a little more today with NeoMinkovskian CONTINUUM which adds a decisive gravitational component ( $G$ - WED) to de Broglie's subquantum substratum [16]. The unified field ( $G-D S R$ is a $G-W E D)$ is photonico-electro-gravific ( $\S$ 5-4).

We suggest here to continue with relativistic mind of de Broglie. The only difference is that we use both $S R$ and $G R$ (and therefore $G$-WED) in order to determine (we put in evidence sub-hypo) the SUB (microphysics quantum stratum).

The most fundamental principle of $Q E D$ (microphysics) is that the LEAST action corresponds to $h$ (or $\hbar$ ). In $G$-WED (Hypomicrophysics) we have the following LEAST action (52):

$$
\left(\frac{e^{2}}{c}\right)_{W E D} \ll \hbar_{Q E D}
$$

the subquantum "continuum" of action $\left(\frac{e^{2}}{c}\right)_{W E D}$, in harmony with continuous spectrum of $C B R$, is smaller $(S U B)$ than the "quantum" of action.

The fine structure constant becomes then a decisive factor between $G$-WED and $Q E D$ in its two forms (Sommerfeld or Planck Einstein):

$$
\frac{\hbar c}{e^{2}} \approx 137, \cdots \quad \frac{h c}{e^{2}} \approx 860, \cdots
$$

In order to treat of (the density of) non-baryonic SUBquantum VACUUM $G$ - WED is then better adapted:

$$
G \text {-WED } D_{\text {Poincare-deBroglie }} \gg Q E D_{\text {Dirac-Feynman }}
$$

Given that we have a direct basic connection (graviton) between electron and photon without the positron (annihilation $e^{+} e^{-}$), Poincaré-de Broglie's G-WED involves then a (cosmological) Break of symmetry (in cosmological observation there is no antimatter, $Q E D)$.

However, as long as we have no precision (56) about $S U B$ formula of density of gravitational waves $(49-3,54)$ exactly as we have density of light waves, this inequality (55) seems purely formal.

\section{Theoretical Deduction of the (Second) Density and the Temperature of CBR}

After the first attempt (42, $\left.w_{e}=w_{e m}\right)$, let us now introduce (second attempt) $w_{G e}=w_{C B R}$ the density of Gravific Waves (53) and then also that of CBR (54 and annex 1):

$$
w_{G e}=\frac{G}{8 \pi} \frac{m_{e}^{2}}{\lambda_{G e}^{4}}=w_{C B R} \simeq 3.8 \times 10^{-34} \mathrm{~g} / \mathrm{cm}^{3}
$$

With Stefan-Boltzman's formula (39, 40 and 80$)$ and numerical annex 1) we 
suggest a theoretical ${ }^{29}$ deduction of the absolute temperature of $C B R\left(R_{H} T_{K}=c t e\right)$ :

$$
T_{K} \approx 2.6 \mathrm{~K}
$$

very close to COBE observation (see annex 1).

The right missing coefficient in (33) (with constant $G$ ) (instead of 48, Poincaré 1905):

$$
p_{G e} \eta_{\mu v}=\frac{1}{3} w_{G e} \eta_{\mu \nu}=\frac{1}{3} \frac{G}{8 \pi} \frac{m_{e}^{2}}{\lambda_{G e}^{4}}\left(\begin{array}{cccc}
1 & 0 & 0 & 0 \\
0 & -1 & 0 & 0 \\
0 & 0 & -1 & 0 \\
0 & 0 & 0 & -1
\end{array}\right)
$$

We can now complete (33):

$$
\begin{aligned}
\rho_{\Lambda} \eta_{\mu v}^{-}=\rho_{\Lambda}\left(\begin{array}{cccc}
-1 & 0 & 0 & 0 \\
0 & 1 & 0 & 0 \\
0 & 0 & 1 & 0 \\
0 & 0 & 0 & 1
\end{array}\right) \text { (dark Energy) } \\
w_{G e} h_{\mu \nu}^{+}=w_{G e}\left(\begin{array}{cccc}
1 & 0 & 0 & 0 \\
0 & -1 & 0 & 0 \\
0 & 0 & -1 & 0 \\
0 & 0 & 0 & -1
\end{array}\right) \quad(\mathrm{CBR})
\end{aligned}
$$

We have not only the density of $C B R$ but also the basic ratio (see 12) with critical density (see anti-32 and 75) directly deducted from NeoMinkowskian limit of $G R$ with $C C$ :

$$
\frac{w_{C B R}}{\rho_{\Lambda}}=\Omega_{C B R}=5.22 \times 10^{-5}=\frac{1}{3}\left(\frac{f_{G e}}{f_{G}}\right)^{2}
$$

(very close to observation $5.38 \times 10^{-5}$ ). The coincidence is obviously not perfect (with the factor $4 / 3$ it's even better, 48 ). For the evaluation of the cosmic substratum density our WED error is of the order 10 to the power -1 . It must be compared (see Unruh) with that of $Q E D$ that is of the order of 10 to the power 120:

"The cosmological constant problem arises because the magnitude of vacuum energy density predicted by quantum mechanics is about 120 orders of magnitude larger than the value implied by observations of accelerating cosmic expansion." [8]

This $C C$ problem reported by Unruh disappears with $W E D$. We prove that his monstrous error comes from the misuse (extrapolation) of baryonic quantum theory to a radically non-baryonic subquantum vacuum (confusion between level 2 and 3 according to de Broglie). Note also that this last ratio (59) is also very close to (53) $\left(\frac{\lambda_{G e}}{\lambda_{e}}\right)^{2} \approx\left(\frac{1}{137}\right)^{2}$.

${ }^{29} \mathrm{No}$ inflationary model predicts such a result (to our knowledge). Flat Cosmology (Tatum) predicts this temperature using not the Planck's particle but Planck's mass. It is very curious (see 83). 


\section{Conclusions: From Cosmological Electron to Galactical Electron?}

Rather than the Quantization of $G R$ (main stream) we chose here the $G R$-ization of Quantum (electron).

The NeoMinkowskian synthesis between Poincaré's GRAVIFIC waves and Poincaré's GRAVIFIC pressure on electron is now complete.

Planck's unstable cosmological particle is then shorted (short-circuited) by stable cosmological electron which is in fact the graviton.

Hyperbolic NeoMinkowskian Universe not only predicts a $C B R$ but also the Temperature observed of this $C B R$.

We follow Penrose [18] on two essential points:

1) "There is something particularly elegant about hyperbolic geometry". $G$-DSR (introduction and $§ 1-2-3$ )

2) "I should say that I do not really believe these (inflationary) theories". $G$-DSR ( $\$ 4$ intermediate conclusions and $\S 5$ conclusions)

The most beautiful result of Lobatchevskian interpretation of "Big Bang" or "Big Boost" may be: The finite time $T_{H}$ is an effect of the curvature of hyperbolic straight line of time $(\S 3-3-3,27)$.

Cosmology joins geology: catastrophism or inflationism (with a Big exceptional Bang of primitive atom) against unifomitarism or gradualism (with Big Well tempered Boost).

We are aware of the incompleteness of this present exploration.

In our approach everything seems RADIAL: there is no RADIAN (no aberration). We have a cosmological electron (Translation) but not a galactical electron (Rotation).

Nothing is circular ( $U C M)$, everything seems rectilinear ( $U A R M$ ). Everything is longitudinal, nothing is transversal: the magnetism seems gone (der verdammte magnetismus, sagte der junge Albert zu Mileva). It could come back in force transversely.

An alternative to the escape velocity (Dark Energy) of this paper however exists: the orbiting velocity (Dark Matter). The only difference is given by a factor $1 / 2$ (in $Y P 12$ ).

The composition of two LT not in the same direction involves Thomas' Rotation (angular velocity connected with frequency of wave electron, 52).

According to Pauli's, Thomas' rotation is a decisive correction of factor $1 / 2$ in Dirac' spin $1 / 2$ of electron.

A Gravitational REVOLUTION in Dirac's electronic spin? De revolutionibus orbium spinorium ...?

(Remember also that all this was done in the absence of baryons $\left(G_{\mu v}=0\right)$ and also especially in the absence of neutrinos).

\section{Special Thanks}

I thank Jean Reignier who oversees my research for 30 years (Université Libre de 
Bruxelles). I thank also P. Marage (idem).

\section{Conflicts of Interest}

The author declares no conflicts of interest regarding the publication of this paper.

\section{References}

[1] Pierseaux, Y. (2014) Journal of Modern Physics, 5, 1725-1732.

http://www.scirp.org/journal/PaperInformation.aspx?PaperID $=51072$

[2] Pierseaux, Y. (2013) Annales de la Fondation Louis de Broglie, 38, 41-55. http://aflb.ensmp.fr/AFLB-381/aflb381m761.pdf

[3] Riess, A., et al. (1998) Astronomical Journal, 116, 1009-1038. https://doi.org/10.1086/300499

[4] Poincaré, H. (1905) Compte Rendus de PAcademie des Sciences de Paris, 140, 1504-1508.

[5] Tatum, E.T. (2019) Journal of Modern Physics, 10, 1083-1089. https://doi.org/10.4236/jmp.2019.109070

[6] Feinberg, G. (1967) Physical Review, 159, 1089-1105. https://doi.org/10.1103/PhysRev.159.1089

[7] Hawking, S. (1988) A Brief History of Time. Bantam Books, Toronto.

[8] Unruh, W.G. (2018) Can the Fluctuations of the Quantum Vacuum Solve the Cosmological Constant Problem? https://arxiv.org/abs/1805.12293

[9] Borel, E. (1913) La théorie de la Relativité et la Cinématique, C.R. Acad. Sci. Paris,

[10] Barrett, J.F. (1993) Lobatchevski, Varicak and Hubble Redshift. European Mathematical Newsletters, March 1993.

[11] Penrose, R. (1997) The Large, the Small and the Human Mind. Cambridge University Press, Cambridge.

[12] Rindler, W. (2001) Relativity. Special, General and Cosmological. Oxford University Press, Oxford.

[13] Pauli, W. (1921) Theory of Relativity. Dover Publications, New York, 1958.

[14] Milgrom, M. (2008) The MOND Paradigme.

[15] Bondi, H. (1962) Relativity and Common Sense. A New Approach to Einstein. Dover Publications Inc., New York.

[16] Pierseaux, Y. (2019) Annales de la Fondation Louis de Broglie, 43, 135-175. http://aflb.ensmp.fr/AFLB-432/aflb432m879.pdf

[17] Landau, L. (1970) Théorie des Champs (avec E. Lifschitz). Editions MIR, Moscou.

[18] Einstein, A. (1905) Annalen der Physik, 17, 891-921. https://doi.org/10.1002/andp.19053221004

[19] Pierseaux, Y. (2000) La “Structure Fine" de la Relativit? Restreinte, L’Harmattan, 1999.

[20] Pierseaux, Y. (2007) L'ellipse de Poincaré, Comptes Rendus Physique, 8, 921-928. http://www.sciencedirect.com/science/article/pii/S1631070507001326 https://doi.org/10.1016/j.crhy.2007.06.002

[21] Reignier, J. (2002) Faut-il croire les cosmologistes? Prof. Em. ULB-VUB. 


\section{Numerical Annex 1: From Cosmological Electron to Gravific Density of CBR}

\subsection{Some Cosmological Constants and Poincaré's Cosmological Electron}

Critic density of tachyonic dark energy from Hubble's constant (1 bis):

$$
\rho_{\Lambda}=\frac{3}{8 \pi} \frac{\left(2.168 \times 10^{-18}\right)^{2}}{6.67428 \times 10^{-8}}=8.6412 \times 10^{-30} \mathrm{~g} / \mathrm{cm}^{3}
$$

Kinematic and Dynamic values: $R_{H}=1.38 \times 10^{28} \mathrm{~cm}$,

$$
\alpha_{M}=7.3543 \times 10^{-8} \mathrm{~cm} / \mathrm{s}^{2}, M_{H}=9.311 \times 10^{55} \mathrm{~g}
$$

with $\left(\rho_{\Lambda}=\frac{\Lambda c^{4}}{8 \pi G}=\frac{3 H_{\Lambda}^{2} c^{2}}{8 \pi G}=\frac{3 \alpha_{M}^{2}}{8 \pi G}=\frac{3 F_{G}}{4 \pi R_{H}^{2}}=\frac{3 G}{2 \pi} \frac{M_{H}^{2}}{R_{H}^{4}}=\frac{3 M_{H} c^{2}}{4 \pi R_{H}^{3}}\right)$.

A coincidence:

$$
\Lambda \cdot M_{H}=10^{-56} \mathrm{~cm}^{-2} \times 10^{56} \mathrm{~g} \approx 1 \mathrm{~g} / \mathrm{cm}^{2}
$$

Enigmatic force between an electronic mass and a cosmological acceleration (Poincaré's force):

$$
f_{G}=m_{e} \alpha_{M}=2 m_{e} G \frac{M_{H}}{R_{H}^{2}}=9.11 \times 10^{-28} \times 7.35 \times 10^{-8}=6.3 \times 10^{-35} \mathrm{cgs}
$$

$f_{G}$ is very close to $f_{G e}(63,31)$.

\subsection{Game of Units (of Thrones?)}

It should not be confused Planck's black body theory $\left(c, k_{B}, \hbar\right)$ with Hypothesis of the so-called Planck's cosmological particle $(c, G, \hbar)$. The latter is an unstable primitive atom (inflation) based on a game of units with 3 constants $(c, G, \hbar)$ (tiny Planck's length $\sqrt{\frac{h G}{c^{3}}}=l_{P l}=10^{-33} \mathrm{~cm}$, huge Planck's density $\rho_{P l}=10^{+94} \mathrm{~g} / \mathrm{cm}^{3}$ and medium Planck's mass $\left.l_{P l} \approx 10^{-5} \mathrm{~g}\right), \quad G=6.67428 \times 10^{-8}$. We have also a game of Stoney's units (i.e. Stoney mass $\sqrt{\frac{e^{2}}{2 G}} \approx 10^{-6} \mathrm{~g}$ ) with also 3 constants $\left(c, G, e^{2} / c\right)$ connected to Planck's units by constant of fine structure $\frac{h c}{e^{2}} \approx 137, \ldots$.

These "games of units" are short-circuited by a basic electronic (or gravific) length $\lambda_{G e}=\frac{e^{2}}{m_{e} c^{2}}=r_{e}=2.8289 \times 10^{-13} \mathrm{~cm}$ and therefore also by the mass of electron $m_{e}=9.109 \times 10^{-28} \mathrm{~g}$ and the charge $e=4.803 \times 10^{-10}$.

The stable cosmological electron (in fact the graviton) is hidden in electro-gravific correspondence (under the presidency of velocity $c$ of light, see $30 \& 32$, $c=2.99792 \times 10^{10} \mathrm{~cm} / \mathrm{s}$ )

$$
c^{2}=\frac{2 G M_{H}}{R_{H}}=\frac{e^{2}}{m_{e} r_{e}}
$$


We have to show that $f_{G}(14,61)$ is perfectly integrated in new (cosmological) theory of unified field.

\subsection{Ratio of Forces}

Next to the macro-force $\left(F_{G}=\frac{c^{4}}{2 G}\right)$ both classical forces respectively electrostatic and gravific $f_{e} \& f_{G e}$,

$$
f_{e}=\frac{e^{2}}{r_{e}^{2}}=2.8826 \times 10^{6} \mathrm{cgs}, \quad f_{\mathrm{Ge}}=\frac{G m_{e}^{2}}{r_{e}^{2}}=6.9216 \times 10^{-37} \mathrm{cgs}
$$

there exists an electrogravific connection (basic constant $\kappa_{G e}$ ) between "infinitely large" ( $M_{H}$ and $\left.R_{H}\right)$ and "infinitely small" $\left(m_{e}\right.$ and $\left.r_{e}\right)$.

$$
\frac{f_{e}}{f_{G e}}=\frac{e^{2}}{G m_{e}^{2}}=\kappa_{G e} \approx 4.1604 \times 10^{42}
$$

We have three forces $\left(F_{G}, f_{e}, f_{G e}\right)$ where $f_{e}$ is well supervised $\left(\kappa_{G e}\right)$ by the other two $f_{e}^{2}=2 F_{G} f_{G e}$ :

$$
\frac{2 F_{G}}{f_{e}}=\frac{f_{e}}{f_{G e}}=\frac{e^{2}}{G m_{e}^{2}}=\kappa_{G e}, \quad \frac{2 F_{G}}{f_{G e}}=\kappa_{G e}^{2}
$$

See $\S 4$ intermediate conclusions:

$$
F_{G} \stackrel{\kappa_{G e}}{\longleftarrow} f_{e} \stackrel{\kappa_{G e}}{\longrightarrow} f_{G e}
$$

At this stage $f_{G}$, very close to $f_{G e}$, is missing (70).

\subsection{Ratio of Lengths and Integration of the Missing Force}

Ratio of linear density respectively for Universe and electron is also given by fundamental physical constant of NeoMinkowskian continuum:

$$
\frac{2 M_{H}}{m_{e}}=\kappa_{G e} \frac{R_{H}}{r_{e}}, \frac{\frac{M_{H}}{R_{H}}}{\frac{m_{e}}{r_{e}}}=\frac{1}{2} \kappa_{G e}
$$

Numerically let us remark that we have approximately $\frac{R_{H}}{r_{e}} \approx \kappa_{G e}$ and then $\frac{2 M_{H}}{m_{e}} \approx \kappa_{G e}^{2}$. More precisely let us introduce $\epsilon$ in such a way that:

$$
\frac{\epsilon R_{H}}{r_{e}}=\kappa_{G e}, \quad \epsilon \approx 90
$$

with the current measures $\epsilon \approx 90$ :

$$
\epsilon=\kappa_{G e} \frac{r_{e}}{R_{H}}=2 \frac{M_{H} r_{e}^{2}}{m_{e} R_{H}^{2}}
$$

Hypothetical "acceleration" $\alpha_{m}=G \frac{m_{e}}{r_{e}^{2}}$ is very close to cosmological accele- 
ration $\alpha_{M}$ :

$$
f_{G}=m_{e} \alpha_{M}=m_{e} 2 G \frac{M_{H}}{R_{H}^{2}}, \quad f_{G e}=m_{e} \frac{G m_{e}}{r_{e}^{2}}=m_{e} \alpha_{m}
$$

Exactly the same ratio $\epsilon$ for the lengths (68):

$$
\frac{\alpha_{M}}{\alpha_{m}}=\frac{f_{G}}{f_{G e}}=2 \frac{M_{H} r_{e}^{2}}{m_{e} R_{H}^{2}}=\epsilon \approx 90
$$

The set of formulas with (61) is therefore perfectly coherent (see the final ratio of densities 49, 72, anti-32):

\subsection{Ratio of Densities}

Leaving aside the first historical attempt (42-43) $w_{\text {Electron }}=\frac{1}{8 \pi} \frac{e^{2}}{r_{e}^{4}}=w_{e} \quad$ (2) with the ratio $\left(\kappa_{G e}=\frac{w_{E l}}{w_{P h}}\right)$, it remains two densities (see anti-32):

$$
\rho_{\text {Tachyon }}=\frac{\Lambda c^{4}}{8 \pi G}=\frac{3 \alpha_{M}^{2}}{8 \pi G}(1) \quad w_{\text {Photon }}=\frac{G}{8 \pi} \frac{m_{e}^{2}}{r_{e}^{4}}=w_{G e}=\frac{1}{8 \pi G} \alpha_{m}^{2}
$$

Our theoretical deduction of the basic (couplage, 12) ratio with $G$-interaction between respectively, "two Universes" and "two Electrons" $\frac{3 G}{2 \pi} \frac{M_{H}^{2}}{R_{H}^{4}}$ and $\frac{G}{8 \pi} \frac{m_{e}^{2}}{r_{e}^{4}}$ :

$$
\frac{\rho_{\Lambda}}{w_{G e}}=\frac{\frac{3 \alpha_{M}^{2}}{8 \pi G}}{\frac{1}{8 \pi G} \alpha_{m}^{2}}=3\left(\frac{\alpha_{M}}{\alpha_{m}}\right)^{2}=3\left(\frac{f_{G}}{f_{G e}}\right)^{2}=3 \epsilon^{2} \approx 19200
$$

Two basic DENSITIES are (density of mass):

$$
\rho_{\Lambda}=\frac{\rho}{c^{2}} \approx 8.6412 \times 10^{-30} \mathrm{~g} / \mathrm{cm}^{3}, \frac{w_{G e}}{c^{2}} \simeq 3.8 \times 10^{-34} \mathrm{~g} / \mathrm{cm}^{3}
$$

The second in details:

$$
\frac{w_{G e}}{c^{2}}=\frac{1}{8 \pi} \times \frac{6.67428 \times 10^{-8} \times\left(9.11 \times 10^{-28}\right)^{2}}{\left(2.8289 \times 10^{-13}\right)^{4} \times\left(2.99 \times 10^{10}\right)^{2}} \approx 3.85 \times 10^{-34} \mathrm{~g} / \mathrm{cm}^{3}
$$

Or the usual inverse ratio:

$$
\frac{w_{C B R}}{\rho_{\Lambda}}=\Omega_{C B R}=5.22 \times 10^{-5}=\frac{1}{3}\left(\frac{f_{G e}}{f_{G}}\right)^{2}
$$

With Stefan-Boltzman's formula with $w_{G e}=w_{C B R}$ we find an absolute temperature of (57).

Observed $C B R(C O B E)$ values:

$$
\begin{gathered}
w_{C O B E}=4.6 \times 10^{-34} \mathrm{~g} / \mathrm{cm}^{3} \\
\frac{\rho_{\Lambda}}{w_{C B R}}=\frac{8.6412 \times 10^{-30}}{4.5908 \times 10^{-34}}=18823
\end{gathered}
$$


or inverted:

$$
\Omega_{C B R}=\frac{w_{C B R}}{\rho_{\Lambda}}=5.38 \times 10^{-5}
$$

Finally let us note that (74-76) are very close to $\left(\frac{1}{137}\right)^{2}$ the ratio (53) between $\lambda_{\text {Poincare }}=\lambda_{G e}$ and $\lambda_{\text {Bohr }}$.

So with $\epsilon=\Omega_{C B R}=1$ it would remain only one "density-pressure":

$$
\rho_{\Lambda}=3 p_{C B R}
$$

Which of the two? Ask the question (between mass of electron and Hubble constant) is answer it. CONJECTURE 1: If our universe $R_{H}$ was about $10^{2}$ larger than the current evaluation from Hubble constant we would have (79). Another CONJECTURE is possible (see annex 2).

\subsection{Law of Stefan: Radiation and Gravitation}

$$
w_{C B R}=\sigma_{\text {Stephan }} T^{4}, \quad \sigma_{\text {Stephan }}=7.56564 \times 10^{-15} \mathrm{cgs}
$$

If we admit (56) we have:

$$
T^{4}=\frac{w_{G e}}{\sigma_{B}}=\frac{G}{8 \pi} \frac{m_{e}^{2}}{r_{e}^{4}} \frac{15 \hbar c^{3}}{8 \pi^{3} k_{B}^{4}}=G \frac{m_{e}^{6} c^{8}}{e^{8}} \frac{15 \hbar^{3} c^{3}}{64 \pi^{4} k_{B}^{4}}=G \frac{m_{e}^{2} m_{e}^{4} c^{8}}{e^{2}} \frac{15\left(\hbar^{3} c^{3}\right)}{64 \pi^{4}\left(e^{6}\right) k_{B}^{4}}
$$

This formula ( $C B H$ or $C B R$ ) might have something to do with Hawking's formula for $S B H$ [9] (especially in his Tatum cosmological interpretation (mean mass) [11]).

$$
\begin{gathered}
\frac{64 \pi^{4}}{15} k_{B}^{4} T^{4}=G \frac{m_{e}^{2}\left(m_{e} c^{2}\right)^{4}}{e^{2}} 137^{3} \Rightarrow \frac{\pi}{15} \kappa_{G e} k_{B}^{4} T^{4}=\left(m_{e} c^{2}\right)^{4} \frac{137^{3}}{4 \pi^{3}} \\
\left(\frac{m_{e} c^{2}}{k_{B} T}\right)^{4}=\frac{\pi}{15} \kappa_{G e}\left(\frac{4 \pi}{137}\right)^{3}
\end{gathered}
$$

The electron is so integrated in $C B R$ and thus also in universal substratum. Observers located inside the universe can be happy they have light with electricity!

\section{Annex 2 Historical Epilogue: The "Fine Structure" of Special Relativity (Poincaré-Einstein, Conjecture 2)}

It seems that the question of the limit $(v=c)$ "Perfectly UltraRelativistic Electron" (PURE) has never been considered in physics.

It's wrong because this question is already considered in 1905 in the two papers of Poincaré and Einstein [4] [18].

We have shown that from a historical point of view there was not only one theory of $S R$ but two theories of $S R$ (quasi-simultaneous, 1905) theories of $S R$ : that of Einstein (June) and that of Poincaré (July).

Both theories are very close but not confused. So there is a "Fine Structure" of $S R$ in epistemological meaning (with quotes [19]). Both theories are based on $L T$ 
and invariance (limit) of light velocity but they are singularities that seemed irreducible: In Poincaré's $S R$ we have for examples: Light elongated ellipsoids $[20]^{30}$, gravific pressure on electron, gravific waves .... In Einstein's $S R$ we have for example: Doppler formula and LichtKomplex. Poincaré's point of view is then very close to NeoMinkoskian Limit of ... Einstein's $G R$.

\subsection{Einstein's "LichtKomplex" and Poincaré's Graviton}

LichtKomplex are introduced by Einstein in June basic paper $\S 8$ [5] three months after his famous LichtQuant (1905). The latter became (with impulsion) the photon for which Einstein got in 1922 the Nobel Prize. Einstein's LichtKomplex has (almost) nothing to do with that.

Einstein's LichtKomplex were considered as horrors (or terrors) by Lorentz, and Planck (and most of physicists). LichtKomplex were rejected by the community of physicists because they presuppose that a certain amount of matter travels at the speed of light. Einstein eliminated them in all subsequent presentations of his $S R$ (already in 1907 ...). Their radical elimination will persist even after 1922. They were ejected from both History of Physics and Physics itself.

Why?

Both they use $L T$ of a spherical volume but Poincaré considers (1905 §1) a sphere driven with an electronic point (invariance of charge and action $e^{2} / c$ ) whilst Einstein's considers (1905 §8) a sphere driven with a photonic point (LichtKomplex). BY MAKING $v=c$ (SIC) in Poincaré's spherical Electron., Einstein deduces the existence of spherical particles "whose energy transforms proportionally the frequency". The coefficient of proportionality is then not $\hbar$ but $e^{2} / c$ (see 52$)$.

Thanks to GR (Einstein 1915) with $C C$ (Einstein 1917) with Poincaré's (NeoMinkowskian) Limit, we now know that when (the young) Einstein makes $v:=c \quad$ (reSIC) in Poincaré's electron, he determines not a photon but a graviton (see 52). The history of physics is highly nonlinear.

Let us finally not that Einstein uses also Langevin's formula (43) for density $(1 / 8 \pi)$, see also Landau 31-5, p106 [17].

\subsection{Poincaré's Velocity with Respect to the Gravific Ether (Conjecture 2)}

The fine structure (without quotes) constant is thus hidden in the synthesis between the two $S R$ (a "Double" $S R$ ). called by Einstein "factor 900 ":

$$
\frac{h}{e^{2} / c} \approx 860
$$

\footnotetext{
${ }^{30}$ Electron, atom, solar system, galaxy are not in expansion. Poincaré could seem "inflationist" at this respect, "Mon lit est en expansion ...”. Et il précise "mais je ne peux pas m’en apercevoir"! Le principe einsteinien (quantique) de l'identité des unités de mesure (des atomes) est à cet égard plus clair. Le fait est que Poincaré avait une théorie de l'expansion (ellipsoédes lumineux allongés, note 18) en 1907 basée sur ... la contraction de Lorentz (20 ans avant l'univers de Hubble.
} 
With Poincaré's [21] (relative) speed with respect to the gravific ether: ${ }^{31}(C B R)$, we have the right to formulate a second conjecture (conjecture of "Big Boost"):

$$
c / 860 \approx 360 \mathrm{~km} / \mathrm{s}
$$

This is very close of the observed $C O B E$ value. A dipolar effect on $3 \mathrm{~K}$. of the order of $3 \mathrm{mK}\left(10^{-3} \approx \frac{1}{900}\right)$. Compatible with the inflationist hot $B B 10^{+3}$ $(3000 \mathrm{~K})$ ?

If we were at rest with respect to the ether, the "quantum" $h$ and the "continuum" $e^{2} / c$ would be thus reconciled.

\footnotetext{
${ }^{31}$ Einstein had suppressed in his $S R$ (1905) the ether (with the possibility of measuring a speed with respect to it). Poincare did not remove the ether because it was a (gravitational) source of the mass of the electron. Note that Einstein reset an ether in 1922 (see L. Kostro) but he could not make the connection with $C B R$ discovered (1965) after his death (1955).
} 